\title{
Charmed-Meson Fragmentation Functions with Finite-Mass Corrections
}

\author{
T. Kneesch ${ }^{1}$, B.A. Kniehl ${ }^{1}$, G. Kramer ${ }^{1}$, and I. Schienbein ${ }^{2}$ \\ ${ }^{1}$ II. Institut für Theoretische Physik, Universität Hamburg, \\ Luruper Chaussee 149, 22761 Hamburg, Germany \\ ${ }^{2}$ Laboratoire de Physique Subatomique et de Cosmologie, Université Joseph Fourier \\ Grenoble 1, CNRS/IN2P3, Institut National Polytechnique de Grenoble, \\ 53 avenue des Martyrs, 38026 Grenoble, France
}

\begin{abstract}
We elaborate the inclusive production of single heavy-flavored hadrons in $e^{+} e^{-}$ annihilation at next-to-leading order in the general-mass variable-flavor-number scheme. In this framework, we determine non-perturbative fragmentation functions for $D^{0}, D^{+}$, and $D^{*+}$ mesons by fitting experimental data from the Belle, CLEO, ALEPH, and OPAL Collaborations, taking dominant electroweak corrections due to photonic initial-state radiation into account. We assess the significance of finite-mass effects through comparisons with a similar analysis in the zero-mass variable-flavor-number scheme.
\end{abstract}

PACS: 12.38.Bx, 12.39.St, 13.66.Bc, 14.40.Lb 


\section{Introduction}

In previous work [1], two of us determined non-perturbative $D^{0}, D^{+}, D^{*+}, D_{s}^{+}$, and $\Lambda_{c}^{+}$fragmentation functions (FFs), both at leading order (LO) and next-to-leading order (NLO) in the modified minimal-subtraction $(\overline{\mathrm{MS}})$ factorization scheme, by fitting the fractional-energy spectra of these hadrons measured by the OPAL Collaboration [2,3] in $e^{+} e^{-}$annihilation on the $Z$-boson resonance at the CERN Large Electron-Positron Collider (LEP1). Apart from untagged cross sections, they also measured the contributions arising from $Z \rightarrow b \bar{b}$ decays. This enabled the authors of Ref. [1 to obtain specific FFs for the transitions $c, b \rightarrow D^{0}, D^{+}, D^{*+}, D_{s}^{+}, \Lambda_{c}^{+}$. The strategy adopted in Ref. [1] was very similar to the one underlying Ref. [4], in which also ALEPH data [5] were fitted, and Ref. 6]. The FFs obtained in Ref. [1] were used as input for a NLO study [7] of charmedmeson hadroproduction in $p \bar{p}$ collisions, which yielded reasonable agreement with data collected by the CDF Collaboration in run II at the Tevatron [8].

Recently, new data on charmed-meson production with much higher accuracy have been presented by the Belle Collaboration [9] at the KEK Asymmetric Electron-Positron Collider for $B$ Physics (KEKB) and the CLEO Collaboration [10] at the Cornell ElectronPositron Storage Ring (CESR). These data offer us the possibility to determine the nonperturbative initial conditions of the FFs much more accurately. Furthermore, the large span in center-of-mass (c.m.) energy $(\sqrt{s})$ ranging from $10.5 \mathrm{GeV}$ [9, 10] way up to 91.2 GeV [2,3,5] provides us with a powerful lever arm to test the Dokshitzer-GribovLipatov-Altarelli-Parisi (DGLAP) [11] evolution of the FFs. These new FFs will enable us to improve our theoretical predictions for the charmed-meson hadroproduction cross sections [7,12] to be compared with the CDF data [8].

The data from Belle and CLEO are located much closer to the thresholds $\sqrt{s}=2 m_{c}$ and $\sqrt{s}=2 m_{b}$ of the transitions $c \rightarrow H_{c}$ and $b \rightarrow H_{b}$, where $H_{c}$ and $H_{b}$ stand for generic $c$ or $b$ hadrons, respectively, than those from ALEPH and OPAL. It might thus be a questionable approximation to treat the partonic cross sections for $e^{+} e^{-} \rightarrow c+X$ and $e^{+} e^{-} \rightarrow b+X$ in the massless approximation, with $m_{c}=m_{b}=0$, as was done in Refs. [1,4,6], where LEP1 data were fitted. Therefore, we take into account the finite quark mass corrections of the form $m^{2} / s\left(m=m_{c}, m_{b}\right)$ in the partonic cross sections to test their significance.

The outline of this paper is as follows. In Section 2, we describe the theoretical formalism of single-hadron inclusive production in $e^{+} e^{-}$annihilation. After reviewing the massless case in Section 2.1, we explain how to include the full mass corrections in Section 2.2. Lengthy expressions are relegated to Appendices $\mathrm{A}$ and $\mathrm{B}$. Specifically, we list the electroweak quark charges in Appendix $\mathrm{A}$ and the mass-dependent coefficient functions in Appendix B. In Section 2.3, we consider the electromagnetic initial-state radiation (ISR) that is inherent to the Belle and CLEO data and explain how to efficiently accommodate the ISR corrections in our fits. In Section 3 , we present several alternative FF sets. They are obtained from global fits to Belle [9], CLEO [10], ALEPH [5], and OPAL [2,3] data, and from separate fits to the $B$-factory (Belle plus CLEO) and $Z$-factory (ALEPH plus 
OPAL) data, both for $m \neq 0$ and for $m=0$. Finally, in Section 4, we present a summary and our conclusions.

\section{Formalism}

We study the inclusive production of a single charmed hadron $H_{c}$, with mass $m_{H}$, in $e^{+} e^{-}$ annihilation via a virtual photon $(\gamma)$ or $Z$ boson,

$$
e^{+}+e^{-} \rightarrow(\gamma, Z) \rightarrow H_{c}+X
$$

where $X$ stands for the residual final state, which goes unobserved. Specifically, we concentrate on the cases $H_{c}=D^{0}, D^{+}, D^{*+}$. In the following, we explain how to calculate the cross section of process (1) at NLO in the parton model of QCD, both in the zeromass (ZM) approach, where all quark masses are neglected, and in the general-mass (GM) approach, where the $c$ and $b$ quarks are taken to be massive. We denote the four-momenta of the virtual gauge boson and the $H_{c}$ hadron by $q$ and $p_{H}$, respectively, so that $s=q^{2}$ and $m_{H}^{2}=p_{H}^{2}$, and introduce the scaling variable $x=2\left(p_{H} \cdot q\right) / q^{2}$. We call the energy of $H_{c}$ and the angle of its three-momentum w.r.t. the beam axis in the c.m. frame $E$ and $\theta$, respectively. Then, $x=2 E / \sqrt{s}$ measures the energy of $H_{c}$ in units of the beam energy.

For unpolarized beams and observed hadrons, the cross section of process (1) at a given value of $\sqrt{s}$ can only depend on $E$ and $\theta$. Since the virtual boson has spin one, the most general form of the differential cross section then reads

$$
\frac{\mathrm{d}^{2} \sigma}{\mathrm{d} x \mathrm{~d} \cos \theta}=\frac{3}{8}\left(1+\cos ^{2} \theta\right) \frac{\mathrm{d} \sigma^{T}}{\mathrm{~d} x}+\frac{3}{4} \sin ^{2} \theta \frac{\mathrm{d} \sigma^{L}}{\mathrm{~d} x}+\frac{3}{4} \cos \theta \frac{\mathrm{d} \sigma^{A}}{\mathrm{~d} x} .
$$

The three terms on the right-hand side are the transverse, longitudinal, and asymmetric contributions, respectively. The first two are associated with the corresponding polarization states of the virtual boson with respect to the direction of the observed hadron. The asymmetric contribution is due to the parity-violating interference terms and is not present in QED. The transverse and longitudinal parts are normalized so that

$$
\frac{\mathrm{d} \sigma}{\mathrm{d} x}=\int_{-1}^{+1} \mathrm{~d} \cos \theta \frac{\mathrm{d}^{2} \sigma}{\mathrm{d} x \mathrm{~d} \cos \theta}=\frac{\mathrm{d} \sigma^{T}}{\mathrm{~d} x}+\frac{\mathrm{d} \sigma^{L}}{\mathrm{~d} x} .
$$

In the parton model, each component $\mathrm{d} \sigma^{P} / \mathrm{d} x(P=T, L)$ on the right-hand side of Eq. (3) can be written, up to power corrections, as a sum of convolutions of partonic cross sections $\mathrm{d} \sigma_{a}^{P}\left(y, \mu, \mu_{f}\right) / \mathrm{d} y$, where $a=g, u, \bar{u}, \ldots, b, \bar{b}$ is the fragmenting parton with fourmomentum $p_{a}, y=2\left(p_{a} \cdot q\right) / q^{2}$, and $\mu$ and $\mu_{f}$ are the renormalization and factorization scales, respectively, with FFs $D_{a}\left(z, \mu_{f}\right)$, where $z=x / y$ is the fraction of energy passed on from parton $a$ to hadron $H_{c}$ in the c.m. frame, as

$$
\frac{\mathrm{d} \sigma^{P}}{\mathrm{~d} x}(x, s)=\sum_{a} \int_{y_{\min }}^{y_{\max }} \frac{\mathrm{d} y}{y} \frac{\mathrm{d} \sigma_{a}^{P}}{\mathrm{~d} y}\left(y, \mu, \mu_{f}\right) D_{a}\left(\frac{x}{y}, \mu_{f}\right)
$$


where the values of $y_{\min }$ and $y_{\max }$ are subject to mass effects to be discussed below. At $\mathrm{NLO}, \mu_{f}$ defines the scale, where the divergence associated with collinear gluon radiation off a massless primary quark or antiquark is to be subtracted.

\subsection{ZM approach}

At NLO in the $\overline{\mathrm{MS}}$ scheme, the cross sections of the relevant partonic subprocesses are given by [13]

$$
\begin{aligned}
& \frac{\mathrm{d} \sigma_{q_{i}}}{\mathrm{~d} y}\left(y, \mu, \mu_{f}\right)=N_{c} \sigma_{0}\left(V_{q_{i}}^{2}+A_{q_{i}}^{2}\right)\left\{\delta(1-y)+\frac{\alpha_{s}(\mu)}{2 \pi}\left[P_{q \rightarrow q}^{(0, T)}(y) \ln \frac{s}{\mu_{f}^{2}}+C_{q}(y)\right]\right\}, \\
& \frac{\mathrm{d} \sigma_{g}}{\mathrm{~d} y}\left(y, \mu, \mu_{f}\right)=2 N_{c} \sigma_{0} \sum_{i=1}^{n_{f}}\left(V_{q_{i}}^{2}+A_{q_{i}}^{2}\right) \frac{\alpha_{s}(\mu)}{2 \pi}\left[P_{q \rightarrow g}^{(0, T)}(y) \ln \frac{s}{\mu_{f}^{2}}+C_{g}(y)\right] .
\end{aligned}
$$

Here, $N_{c}=3$ is the number of quark colors;

$$
\sigma_{0}=\frac{4 \pi \alpha^{2}}{3 s}
$$

with $\alpha$ being Sommerfeld's fine-structure constant, is the total cross section of $e^{+} e^{-} \rightarrow$ $\mu^{+} \mu^{-}$for massless muons; $V_{q_{i}}$ and $A_{q_{i}}$ are the effective vector and axial-vector couplings of quark $q_{i}$ to the photon and the $Z$ boson including propagator adjustments, which are listed in Appendix [A: $P_{a \rightarrow b}^{(0, T)}$ are the LO timelike splitting functions (see, e.g., Ref. [14]),

$$
\begin{aligned}
& P_{q \rightarrow q}^{(0, T)}(y)=C_{F}\left[\frac{3}{2} \delta(1-y)+\frac{1+y^{2}}{(1-y)_{+}}\right], \\
& P_{q \rightarrow g}^{(0, T)}(y)=C_{F} \frac{1+(1-y)^{2}}{y}
\end{aligned}
$$

and the coefficient functions read [13]

$$
\begin{aligned}
C_{q}(y)= & C_{F}\left\{\left(-\frac{9}{2}+\frac{2}{3} \pi^{2}\right) \delta(1-y)-\frac{3}{2}\left(\frac{1}{1-y}\right)_{+}+2\left[\frac{\ln (1-y)}{1-y}\right]_{+}+\frac{5}{2}-\frac{3}{2} y\right. \\
& \left.+4 \frac{\ln y}{1-y}-(1+y)[2 \ln y+\ln (1-y)]\right\}, \\
C_{g}(y)= & C_{F} \frac{1+(1-y)^{2}}{y}[2 \ln y+\ln (1-y)],
\end{aligned}
$$

where $C_{F}=\left(N_{c}^{2}-1\right) /\left(2 N_{c}\right)=4 / 3$ and the plus distributions are defined as usual. We evaluate the strong-coupling constant $\alpha_{s}$ using the two-loop formula with $n_{f}=5$ quark flavors. We identify $\mu=\mu_{f}=\sqrt{s}$, so that in Eq. (5) the terms proportional to $\ln \left(s / \mu_{f}^{2}\right)$ vanish. We observe that $C_{g}(y)<0$ for any value of $y$, so that the gluon contributes 
destructively to Eq. (5). The bounds of integration in Eq. (44) are $y_{\min }=x, y_{\max }=1$, and we have $\sqrt{\rho_{H}} \leq x \leq 1$, where $\rho_{H}=4 m_{H}^{2} / s$.

For later use, we also list the total hadronic cross section at NLO,

$$
\sigma_{\text {tot }}=N_{c} \sigma_{0} \sum_{i=1}^{n_{f}}\left(V_{q_{i}}^{2}+A_{q_{i}}^{2}\right)\left[1+\frac{\alpha_{s}(\mu)}{2 \pi} C_{F} \frac{3}{2}\right] \text {. }
$$

The $x$ dependences of the FFs are not yet calculable from first principles. However, once they are given at some initial fragmentation scale $\mu_{0}$, their $\mu_{f}$ evolution is determined by the DGLAP evolution equations [11],

$$
\frac{\mathrm{d}}{\mathrm{d} \ln \mu_{f}^{2}} D_{a}\left(x, \mu_{f}\right)=\frac{\alpha_{s}(\mu)}{2 \pi} \sum_{b} \int_{x}^{1} \frac{\mathrm{d} y}{y} P_{a \rightarrow b}^{T}\left(y, \alpha_{s}(\mu)\right) D_{b}\left(\frac{x}{y}, \mu_{f}\right) .
$$

Specifically, we use $\mu_{0}=m_{c}$ for $a=g, u, \bar{u}, d, \bar{d}, s, \bar{s}, c, \bar{c}$ and $\mu_{0}=m_{b}$ for $a=b, \bar{b}$. Our task is thus to construct a model for the $z$ dependences of $D_{a}\left(z, \mu_{0}\right)$, which upon evolution to $\mu_{f}=\sqrt{s}$ fit the data at that c.m. energy.

The above formalism is identical to the one that is routinely used in the literature for the inclusive production of single light hadrons [15. The non-zero values of the $c$ - and $b$-quark masses only enter through the initial conditions of the FFs, and the mass of the heavy hadron sets the lower bound on the scaling variable $x$.

\subsection{GM approach}

We derived the partonic cross sections at NLO for non-zero quark masses adopting the on-shell definition of the latter and found agreement with Ref. [16]. We take the pole masses of the $c$ and $b$ quarks to be $m_{c}=1.5 \mathrm{GeV}$ and $m_{b}=5.0 \mathrm{GeV}$, respectively. The finite-mass corrections are generally of order $m^{2} / s\left(m=m_{c}, m_{b}\right)$. They can be sizeable for sufficiently small values of $\sqrt{s}$. In our study, where the smallest value of $\sqrt{s}$ is $10.52 \mathrm{GeV}$, they reach at least $2 \%$ for the $c$ quark, but might be much larger depending on the coefficient of $\mathrm{m}^{2} / \mathrm{s}$ in the LO cross section. On the other hand, they are quite substantial for the $b$ quark at values of $\sqrt{s}$ just above the $b \bar{b}$ production threshold.

In the following, we consider finite-mass effects of order $\mathrm{m}^{2} / \mathrm{s}$ only in the production dynamics, but not in the decays of the produced $H_{c}$ mesons, which are affected by kinematic power corrections. This would be beyond the scope of our analysis and is not required because the experimental analyses actually provide production cross sections.

In order to expose the connection with the ZM limit and to establish the finite subtraction terms, which are needed for the evaluation of the GM cross section in the $\overline{\mathrm{MS}}$ factorization scheme, we report the relevant cross section formulae for the GM case. We largely adopt the notation of Ref. [16]. 
At NLO, there is a contribution from real gluon radiation. The gluon can either be included in the hadronic system $X$ or it can act as the leading parton initiating a hadron jet that contains the $H_{c}$ meson. Therefore, we also need to consider at the parton level single-gluon inclusive production with $X$ including a $c \bar{c}$ or $b \bar{b}$ pair.

The partonic cross sections of single-heavy-quark inclusive production read

$$
\frac{\mathrm{d} \sigma_{q_{i}}^{P}}{\mathrm{~d} y}(y, \mu)=N_{c} \sigma_{0}\left[V_{q_{i}}^{2} F_{P}^{(v)}(y, \rho)+A_{q_{i}}^{2} F_{P}^{(a)}(y, \rho)\right],
$$

where $\rho=4 m^{2} / s$ with $m=m_{c}, m_{b}$. Notice that the vector and axial-vector contributions differ by finite-mass terms. At NLO, the coefficient functions $F_{P}^{(u)}(y, \rho)$, with $P=T, L$ and $u=v, a$, in Eq. (11) may be decomposed as [16]:

$$
F_{P}^{(u)}(y, \rho)=\delta(1-y) B_{P}^{(u)}(\rho)+\frac{\alpha_{s}(\mu)}{2 \pi}\left[\delta(1-y) S_{P}^{(u)}(\rho)+\left(\frac{1}{1-y}\right)_{+} R_{P}^{(u)}(y, \rho)\right] .
$$

The LO terms read

$$
B_{T}^{(v)}(\rho)=\beta, \quad B_{L}^{(v)}(\rho)=\frac{\rho \beta}{2}, \quad B_{T}^{(a)}(\rho)=\beta^{3}, \quad B_{L}^{(a)}(\rho)=0,
$$

where $\beta=\sqrt{1-\rho}$. In the massless limit, we have $B_{T}^{(v)}(\rho)=B_{T}^{(a)}(\rho)=1$ and $B_{L}^{(v)}(\rho)=$ $B_{L}^{(a)}(\rho)=0$. The NLO terms, $S_{P}^{(u)}(\rho)$ and $R_{P}^{(u)}(y, \rho)$, may be found in Appendix $\mathbb{B}$. The partonic cross section of single-gluon inclusive production reads

$$
\frac{\mathrm{d} \sigma_{g}^{P}}{\mathrm{~d} y}(y, \mu)=N_{c} \sigma_{0} \frac{\alpha_{s}(\mu)}{2 \pi}\left[V_{q_{i}}^{2} G_{P}^{(v)}(y, \rho)+A_{q_{i}}^{2} G_{P}^{(a)}(y, \rho)\right],
$$

with the coefficient functions $G_{P}^{(u)}(y, \rho)$ given in Appendix B. Deviating from the notation of Ref. [16], we included the factor $C_{F}$ in the functions $S_{P}^{(u)}(\rho), R_{P}^{(u)}(y, \rho)$, and $G_{P}^{(u)}(y, \rho)$ to simplify the comparison with the ZM approximation. Through this comparison, we recover the so-called perturbative FFs [17], from which the subtraction terms for the conversion from the NLO calculation with finite quark masses in the on-shell scheme [16] to the GM variable-flavor number scheme [7,12] are constructed. The result thus obtained contains all the finite-mass terms and, at the same time, smoothly approaches the ZM result in the limit $m \rightarrow 0$.

Thanks to our specific choice of hadronic and partonic scaling variables, $x$ and $y$, respectively, Eq. (4) applies to the GM case as it stands. The bounds of integration in $y$ and the allowed $x$ range now depend on the partonic subprocess and on the fragmenting parton $a$. In the case of heavy-quark fragmentation, we have $y_{\min }=\max (x, \sqrt{\rho}), y_{\max }=1$, and $\sqrt{\rho_{H}} \leq x \leq 1$. If a gluon fragments and there is also a heavy-quark pair in the final state, then we have $y_{\min }=x, y_{\max }=\beta^{2}$, and $\sqrt{\rho_{H}} \leq x \leq \beta^{2}$. If there are only massless partons in the final state, then we have $y_{\min }=x, y_{\max }=1$, and $\sqrt{\rho_{H}} \leq x \leq 1$ as in Section 2.1.

The experimental data at $\sqrt{s}=m_{Z}$, collected by OPAL [2]3 and ALEPH [5] at LEP1, come in the form $\mathrm{d} \sigma / \mathrm{d} x$ as a function of the scaling variable $x$ introduced above. As 
explained above, the maximum $x$ range is $\sqrt{\rho_{H}} \leq x \leq 1$. On the other hand, Belle 9] and CLEO [10] present their data as distributions $\mathrm{d} \sigma / \mathrm{d} x_{p}$ in the scaled momentum $x_{p}=p / p_{\max }=\sqrt{\left(x^{2}-\rho_{H}\right) /\left(1-\rho_{H}\right)}$, with allowed values $0 \leq x_{p} \leq 1$. The conversion formula reads

$$
\frac{\mathrm{d} \sigma}{\mathrm{d} x_{p}}\left(x_{p}\right)=\left(1-\rho_{H}\right) \frac{x_{p}}{x} \frac{\mathrm{d} \sigma}{\mathrm{d} x}(x),
$$

with $x=\sqrt{\left(1-\rho_{H}\right) x_{p}^{2}+\rho_{H}}$.

We conclude this section with a remark concerning parton-model kinematics of fragmentation in the presence of finite quark and hadron masses. In the picture where the fragmenting parton $a$ creates a jet that includes the observed hadron $H$, the parton virtuality $p_{a}^{2}$ must exceed the hadron mass square $m_{H}^{2}$. In fact, if the scaling variable $z$ is defined in terms of light-cone momenta, as $z=p_{H}^{+} / p_{a}^{+}=\left(p_{H}^{0}+p_{H}^{3}\right) /\left(p_{a}^{0}+p_{a}^{3}\right)$ [18], we have $p_{a}^{2}>m_{H}^{2} / z$ [19]. Since the outgoing partons are taken to be on-shell in the parton model, this inequality becomes $m^{2}>m_{H}^{2} / z$. In our case, this is only satisfied for the transition $b \rightarrow H_{c}$ in the constrained $z$ range $m_{H}^{2} / m^{2}<z<1$.

At this point, we find find it instructive to insert a digression on the massive kinematics of fragmentation, a topic which has received very little attention in the literature. Let us view the fragmentation process $b \rightarrow H_{c}$ as a decay $b \rightarrow H_{c}+X$, where the invariant mass $m_{X}$ of the hadronic system $X$, which we may treat as one effective particle, can be tuned in the range $0 \leq m_{X} \leq m-m_{H}$. Starting from the rest frame of the decay, we perform a Lorentz boost along the three-momentum of $H_{c}$, such that the energy of $H_{c}$ becomes $p_{H}^{0}=x \sqrt{s} / 2$, where $\sqrt{s}$ is the $e^{+} e^{-}$c.m. energy. This corresponds to the situation where $H_{c}$ is emitted collinearly from $b$, carrying energy $p_{b}^{0}=y \sqrt{s} / 2$, in the laboratory frame. For given values of $x$ and $m_{X}$, we have

$$
y=\frac{1}{2 m_{H}^{2}}\left[\left(m^{2}+m_{H}^{2}-m_{X}^{2}\right) x-\sqrt{\lambda\left(m^{2}, m_{H}^{2}, m_{X}^{2}\right)\left(x^{2}-\rho_{H}\right)}\right],
$$

where $\lambda(a, b, c)=a^{2}+b^{2}+c^{2}-2(a b+b c+c a)$. Owing to the constraint $\sqrt{\rho} \leq y \leq 1$ on $y$ and the one on $m_{X}$ specified above, $x$ must lie in the range $\sqrt{\rho_{H}} \leq x \leq x_{\max }$, with

$$
x_{\max }=\frac{1}{2 m^{2}}\left[m^{2}+m_{H}^{2}+\beta\left(m^{2}-m_{H}^{2}\right)\right] .
$$

For a given value of $x$, the allowed $y$ range is thus $y_{\min }(x) \leq y \leq y_{\max }(x)$, where

$$
\begin{aligned}
& y_{\min }(x)= \begin{cases}\sqrt{\rho} & \text { if } \sqrt{\rho_{H}} \leq x \leq \frac{m^{2}+m_{H}^{2}}{m \sqrt{s}} \\
y_{0} \quad \text { if } \frac{m^{2}+m_{H}^{2}}{m \sqrt{s}}<x \leq x_{\max }\end{cases} \\
& y_{\max }(x)= \begin{cases}\min \left(y_{0}, 1\right) & \text { if } \sqrt{\rho_{H}} \leq x \leq\left(m+m_{H}\right) \sqrt{\frac{m_{H}}{m s}} \\
\min \left(x \frac{m}{m_{H}}, 1\right) & \text { if }\left(m+m_{H}\right) \sqrt{\frac{m_{H}}{m s}}<x \leq x_{\max }\end{cases}
\end{aligned}
$$

where

$$
y_{0}=\frac{1}{2 m_{H}^{2}}\left[\left(m^{2}+m_{H}^{2}\right) x-\left(m^{2}-m_{H}^{2}\right) \sqrt{x^{2}-\rho_{H}}\right] .
$$


For $\sqrt{s} \geq\left(m^{2}+m_{H}^{2}\right) / m_{H}$, the scaling variable $z=x / y$ appearing in the $b \rightarrow H_{c}$ FF lies in the range $2 m_{H}^{2} /\left(m^{2}+m_{H}^{2}\right) \leq z \leq x_{\max }$.

If $m_{H} \ll m$ is a good approximation, we may simplify these expressions by putting $m_{H}=0$. Then, we have $0 \leq x \leq x_{\max }$, with $x_{\max }=(1+\beta) / 2$, and

$$
\begin{aligned}
& y_{\min }(x)=\left\{\begin{array}{ll}
\sqrt{\rho} & \text { if } 0 \leq x \leq \frac{\sqrt{\rho}}{2} \\
x+\frac{\rho}{4 x} & \text { if } \frac{\sqrt{\rho}}{2}<x \leq x_{\max }
\end{array},\right. \\
& y_{\max }(x)=1
\end{aligned}
$$

Consequently, we have $0 \leq z \leq x_{\max }$. Finally, if also $m=0$, we recover the ZM situation, $0 \leq x \leq 1$ and $x \leq y \leq 1$, so that $0 \leq z \leq 1$.

In Fig. 1, the kinematically accessible region in the $(x, y)$ plain is shown for our fragmentation model of $b \rightarrow H_{c}$ assuming LEP1 experimental conditions, with $\sqrt{s}=m_{Z}$, $m=5.0 \mathrm{GeV}$, and $m_{H}=2.01 \mathrm{GeV}$ (solid line). It does not exhaust the region encompassed by $\sqrt{\rho_{H}} \leq x \leq 1$ and $\max (x, \sqrt{\rho}) \leq y \leq 1$ that we adopt for the evaluation of Eq. (4) in the case of $b \rightarrow H_{c}$ (dashed line). There is a wedge missing at small values of $x$ and large values of $y$, i.e. small values of $z$. The minimum value of $z$, $2 m_{H}^{2} /\left(m^{2}+m_{H}^{2}\right) \approx 0.28$, is reached at the minimum value of $x$. However, in the bulk of the missing wedge, we have $z \leq m_{H} / m \approx 0.40$. As we shall see in Section 3 , this excluded $z$ range actually accommodates the peak of the $b \rightarrow H_{c}$ FF (see also Table 8). Moreover, our simple fragmentation picture cannot describe the transitions $a \rightarrow H_{c}$ for $a=u, d, s, c, g$. For these reasons, we abandon it at this point. A crucial conceptual drawback of the parton model applied to the inclusive production of heavy hadrons is that fragmenting partons are taken to be on-shell. We escape this problem by imagining that the hadronic system initiated by the fragmenting parton $a$ not only receives color, but also energy and momentum from the rest of the event. In this way, the accessible region in the $(x, y)$ plane is expanded to become the one underlying our analysis.

\subsection{Electromagnetic initial-state radiation}

The cross sections of inclusive single-hadron production in $e^{+} e^{-}$annihilation measured by Belle [9], CLEO [10], ALEPH [5], and OPAL [2,3] naturally include electroweak corrections, which were not subtracted in the data analyses. The bulk of these corrections is due to the effect of electromagnetic radiation emitted from the incoming electrons and positrons. This ISR is suppressed by a factor of $\alpha$, but enhanced by the large logarithm $\log \left(s / m_{e}^{2}\right)$, where $m_{e}$ is the electron mass. At Belle and CLEO energies, the hadronic cross section decreases with increasing invariant mass of the hadronic system. Since ISR reduces the hadronic mass, it leads to an increase in cross section. The shape of the FF is also changed, since a fraction of the events takes place at lower hadronic invariant mass. The impact of ISR on the determination of FFs has already been considered in Ref. [20] and has been found to be non-negligible for the analysis of the Belle and CLEO data. 
The most straightforward way to correct for ISR would be to incorporate into the general expression for the $e^{+} e^{-} \rightarrow H_{c}+X$ cross section without photon radiation, presented in Section 2.1, the corrections due to photon radiation off the initial-state leptons and use the resulting expression to fit the FFs to the Belle and CLEO data. This procedure would involve several additional numerical integrations in each iteration of the fitting procedure and thus dramatically slow down the latter. In the following, we explicitly derive an approximation formula, which reduces the number of integrations to a manageable level and is still rather precise. Our procedure differs from the one used in Ref. [20], where the ISR corrections were subtracted in an iterative way from the experimental data before the actual fit. Our procedure is numerically more involved, but offers the advantage that, at at the end, the ISR corrections precisely refer to the final $x$ distribution resulting from the fit.

The dominant ISR corrections are conveniently incorporated using the structure-function approach, in which the photon emission is taken to be collinear to the incoming $e^{ \pm}$leptons [21,22]. In analogy to the factorization formula of the collinear parton model, the ISRcorrected differential cross section $d \sigma_{\mathrm{ISR}}\left(p_{+}, p_{-}\right)$, where $p_{ \pm}$denote the four-momenta of the incoming $e^{ \pm}$leptons, is obtained by convoluting the uncorrected differential cross section $d \sigma\left(p_{+}, p_{-}\right)$with radiator functions $D_{e^{ \pm}}\left(x_{ \pm}, s\right)$, one for each incoming leg, which measure the probabilities for the $e^{ \pm}$leptons to retain the fractions $x_{ \pm}$of their energies after the emission of ISR, as

$$
\mathrm{d} \sigma_{\mathrm{ISR}}\left(p_{+}, p_{-}\right)=\int_{0}^{1} \mathrm{~d} x_{+} \int_{0}^{1} \mathrm{~d} x_{-} D_{e^{+}}\left(x_{+}, s\right) D_{e^{-}}\left(x_{-}, s\right) \mathrm{d} \sigma\left(x_{+} p_{+}, x_{-} p_{-}\right) .
$$

Using the method by Gribov and Lipatov [23], the leading logarithms can be resummed to all orders, leading to the expression for $D_{e^{ \pm}}(x, s)$ in Eq. (7) of Ref. [22]. The structurefunction approach was mostly applied to total cross sections in the literature. In this case, one integration can be carried out independently of the considered process and leads to the luminosity function

$$
H_{e^{+} e^{-}}(\tau, s)=\int_{0}^{1} \mathrm{~d} x_{+} \int_{0}^{1} \mathrm{~d} x_{-} \delta\left(\tau-x_{+} x_{-}\right) D_{e^{+}}\left(x_{+}, s\right) D_{e^{-}}\left(x_{-}, s\right)
$$

in $\tau=x_{+} x_{-}$. The Gribov-Lipatov-resummed expression for it may be found in Eqs. (8) and (9) of Ref. [22], in terms of the variable $\chi=1-\tau$. However, if the cross section is differential w.r.t. variables, whose differentials are not invariant under boosts along the beam axis, as in our case, the situation is more involved. In this case, we obtain from Eq. (21) the following master formula:

$$
\begin{aligned}
\frac{\mathrm{d} \sigma_{\text {ISR }}}{\mathrm{d} x}(x, s)= & \int \mathrm{d} x_{+} \mathrm{d} x_{-} \mathrm{d} x^{\prime} \mathrm{d} \cos \theta^{\prime} \delta\left(x-x\left(x_{+}, x_{-}, x^{\prime}, \cos \theta^{\prime}\right)\right) D_{e^{+}}\left(x_{+}, s\right) D_{e^{-}}\left(x_{-}, s\right) \\
& \times \frac{\mathrm{d}^{2} \sigma}{\mathrm{d} x^{\prime} \mathrm{d} \cos \theta^{\prime}}\left(x^{\prime}, \cos \theta^{\prime}, x_{+} x_{-} s\right)
\end{aligned}
$$


where the primed variables refer to the hadronic c.m. frame, which is reached from the $e^{+} e^{-}$c.m. frame through a Lorentz boost with velocity $\tilde{\beta}=\left(x_{+}-x_{-}\right) /\left(x_{+}+x_{-}\right)$, and

$$
x\left(x_{+}, x_{-}, x^{\prime}, \cos \theta^{\prime}\right)=\tilde{\gamma}\left(\sqrt{\tau} x^{\prime}+\tilde{\beta} \sqrt{\tau x^{\prime 2}-\rho_{H}} \cos \theta^{\prime}\right),
$$

with $\tilde{\gamma}=1 / \sqrt{1-\tilde{\beta}^{2}} 1$ Integrating Eq. (23) over $\cos \theta^{\prime}$, we have

$$
\begin{aligned}
\frac{\mathrm{d} \sigma_{\mathrm{ISR}}}{\mathrm{d} x}(x, s)= & \int_{x}^{1} \mathrm{~d} x^{\prime}\left(\int_{x / x^{\prime}}^{1} \mathrm{~d} x_{+} \int_{a_{-} /\left(x^{\prime}-a_{+} / x_{+}\right)}^{a_{+} /\left(x^{\prime}-a_{-} / x_{+}\right)} \mathrm{d} x_{-}+\int_{x / x^{\prime}}^{1} \mathrm{~d} x_{-} \int_{a_{-} /\left(x^{\prime}-a_{+} / x_{-}\right)}^{a_{+} /\left(x^{\prime}-a_{-} / x_{-}\right)} \mathrm{d} x_{+}\right) \\
& \times \frac{2 D_{e^{+}}\left(x_{+}, s\right) D_{e^{-}}\left(x_{-}, s\right)}{\left|x_{+}-x_{-}\right| \sqrt{x^{\prime 2}-\rho_{H} / \tau}} \frac{\mathrm{d}^{2} \sigma}{\mathrm{d} x^{\prime} \mathrm{d} \cos \theta^{\prime}}\left(x^{\prime}, \frac{2 x-\left(x_{+}+x_{-}\right) x^{\prime}}{\left(x_{+}-x_{-}\right) \sqrt{x^{\prime 2}-\rho_{H} / \tau}}, \tau s\right),
\end{aligned}
$$

where

$$
a_{ \pm}=\frac{x \pm \sqrt{x^{2}-\rho_{H}}}{2} .
$$

Notice that the second pair of integrations enclosed within the parentheses in Eq. (25) merely duplicates the first one if $D_{e^{+}}(x, s)=D_{e^{-}}(x, s)$.

Detailed numerical inspection of our specific application reveals that, to very good approximation, we may substitute in Eq. (25)

$$
D_{e^{+}}\left(x_{+}, s\right) D_{e^{-}}\left(x_{-}, s\right) \approx \delta\left(1-x_{+}\right) H_{e^{+} e^{-}}\left(x_{-}, s\right),
$$

which trivially satisfies Eq. (22), so as to eliminate the second term within the parentheses in Eq. (25) and to save one integration in the first one. This implies that the whole ISR is emitted by the electron alone, while the positron stays idle, or vice versa. A further simplification may be obtained by casting Eq. (25) into a form that contains on the r.h.s. $\left(\mathrm{d} \sigma / \mathrm{d} x^{\prime}\right)\left(x^{\prime}, s\right)$, i.e. the cross section at the $e^{+} e^{-}$c.m. energy integrated over the polar angle. To this end, we use the approximations

$$
\begin{aligned}
\frac{\mathrm{d}^{2} \sigma}{\mathrm{d} x^{\prime} \mathrm{d} \cos \theta^{\prime}}\left(x^{\prime}, \cos \theta^{\prime}, \tau s\right) & \approx \frac{3}{8}\left(1+\cos ^{2} \theta^{\prime}\right) \frac{\mathrm{d} \sigma}{\mathrm{d} x^{\prime}}\left(x^{\prime}, \tau s\right) \\
& \approx \frac{3}{8}\left(1+\cos ^{2} \theta^{\prime}\right) \frac{\sigma(\tau s)}{\sigma(s)} \frac{\mathrm{d} \sigma}{\mathrm{d} x^{\prime}}\left(x^{\prime}, s\right) .
\end{aligned}
$$

The approximation in the first line of Eq. (28) may be justified by observing that, in Eq. (2), $\mathrm{d} \sigma^{L} / \mathrm{d} x$ is suppressed for $m^{2} \ll s$, being zero at LO in the ZM approach, and that the contribution from $\mathrm{d} \sigma^{A} / \mathrm{d} x$ vanishes upon integration over $\cos \theta^{\prime}$. The approximation in the second line of Eq. (28) faithfully describes the leading power-like dependence on the c.m. energy, but disregards the scaling violations of the FFs, which are just logarithmic.

\footnotetext{
${ }^{1}$ We denote the relativistic boost velocity and its $\gamma$ factor by $\tilde{\beta}$ and $\tilde{\gamma}$, respectively, because $\beta$ and $\gamma$ are reserved for other quantities in this paper.
} 
For simplicity, we evaluate the factor $\sigma(\tau s) / \sigma(s)$ in Eq. (28) at LO in the GM approach neglecting the contribution from $Z$-boson exchange, as

$$
\frac{\sigma(\tau s)}{\sigma(s)}=\frac{1}{\tau} \frac{1+\rho /(2 \tau)}{1+\rho / 2} \sqrt{\frac{1-\rho / \tau}{1-\rho}} .
$$

On the one hand, the NLO corrections largely cancel out in this cross section ratio; on the other hand, the $Z$-boson contribution is suppressed for $\sqrt{s} \ll m_{Z}$, as explained in Appendix A, Substituting Eqs. (27) and (28) into Eq. (25), we obtain our working formula

$$
\frac{\mathrm{d} \sigma_{\mathrm{ISR}}}{\mathrm{d} x}(x, s) \approx \int_{x}^{1} \mathrm{~d} x^{\prime} \frac{\mathrm{d} \sigma}{\mathrm{d} x^{\prime}}\left(x^{\prime}, s\right) \int_{a_{-} /\left(x^{\prime}-a_{+}\right)}^{a_{+} /\left(x^{\prime}-a_{-}\right)} \mathrm{d} \tau \frac{2 H_{e^{+} e^{-}}(\tau, s)}{(1-\tau) \sqrt{x^{\prime 2}-\rho_{H} / \tau}} \frac{3}{8}\left(1+\cos ^{2} \theta^{\prime}\right) \frac{\sigma(\tau s)}{\sigma(s)}
$$

where

$$
\cos \theta^{\prime}=\frac{2 x-(1+\tau) x^{\prime}}{(1-\tau) \sqrt{x^{\prime 2}-\rho_{H} / \tau}}
$$

and $\sigma(\tau s) / \sigma(s)$ is given in Eq. (29). In Eq. (30), we insert the NLO expression for $\left(\mathrm{d} \sigma / \mathrm{d} x^{\prime}\right)\left(x^{\prime}, s\right)$. Strictly speaking, we should then also evaluate Eq. (29) at NLO. However, the omitted correction is insignificant, as explained below Eq. (29). We employ this formalism also in the ZM approach, except that we then set $\rho=0$ in Eq. (29) for consistency.

The effect of ISR on the $x_{p}$ distribution of $e^{+} e^{-} \rightarrow D^{+}+X$ measured by Belle [9] and CLEO [10] is studied for the GM approach in Fig. 2, where the result of the ISR-corrected fit to these data is compared with the corresponding result where the ISR corrections are subtracted. As already observed in Ref. [20], we find that the spectrum is shifted to larger values of $x_{p}$, i.e. it becomes harder and lower at the peak when the ISR corrections are subtracted. This is expected, since ISR reduces the available hadronic c.m. energy, which softens the spectrum and increases the cross section.

At LEP1 energy, the shift of the spectrum due to ISR is negligible. This may be understood by observing that ISR shifts the c.m. energy available for the hard scattering to values below the $Z$-boson resonance, where the cross section is greatly reduced. In the analysis described below, we thus only include ISR corrections in our theoretical description of the Belle [9] and CLEO [10] data, while we neglect them in connection with the ALEPH [5] and OPAL [2,3] data.

\section{Results}

As experimental input for our fits, we use the $x_{p}$ distributions of $D^{0}, D^{+}$, and $D^{*+}$ production in the continuum at $\sqrt{s}=10.52 \mathrm{GeV}$ from Belle [9] and CLEO [10], which we correct for ISR as described in Section 2.3, and the $x$ distributions of $D^{0}, D^{+}[2]$, and $D^{*+}$ 
[3.5] production on the $Z$-boson resonance at $\sqrt{s}=91.2 \mathrm{GeV}$ from ALEPH and OPAL. We received the Belle data in numerical form via private communication [24]. Belle [9] also provide data from the $\Upsilon(5 S)$ resonance outside the $B$-meson decay region, which we leave aside.

In Refs. 2, 3,5, the absolute cross section distributions in $x$ are normalized to the total hadronic cross section and include the branching fractions of the decays used to identify the $H_{c}$ mesons, namely $D^{0} \rightarrow K^{-} \pi^{+}, D^{+} \rightarrow K^{-} \pi^{+} \pi^{+}$, and $D^{*+} \rightarrow D^{0} \pi^{+}$followed by $D^{0} \rightarrow K^{-} \pi^{+}$, respectively. Therefore, we multiply our predictions by $1 / \sigma_{\text {tot }}$, where, for simplicity, we evaluate $\sigma_{\text {tot }}$ from Eq. (9) exploiting the insignificance of quark mass effects at LEP1 energy, and divide the experimental data by the respective decay branching fractions. For consistency, we adopt the very values of the latter that are used in Refs. [2,3,5]. These read $B\left(D^{0} \rightarrow K^{-} \pi^{+}\right)=(3.84 \pm 0.13) \%$ and $B\left(D^{+} \rightarrow K^{-} \pi^{+} \pi^{+}\right)=(9.1 \pm 0.6) \%$ [25] for Ref. [2], $B\left(D^{*+} \rightarrow D^{0} \pi^{+}\right)=(68.3 \pm 1.4) \%$ and $B\left(D^{0} \rightarrow K^{-} \pi^{+}\right)=(3.83 \pm 0.12) \%$ [26] for Ref. [3], and $B\left(D^{*+} \rightarrow D^{0} \pi^{+}\right)=(68.3 \pm 1.4) \%$ and $B\left(D^{0} \rightarrow K^{-} \pi^{+}\right)=(3.85 \pm 0.09) \%$ [27] for Ref. [5], respectively. The $b$-tagged samples of Refs. [2,3] are treated in the same way as the full cross sections, while the one in Ref. [5] needs to be multiplied by $R_{b} f\left(b \rightarrow D^{* \pm}\right)$, where $R_{b}=\Gamma(Z \rightarrow b \bar{b}) / \Gamma(Z \rightarrow$ hadrons $)$ is the fraction of $b$-tagged events in the full hadronic sample and $f\left(b \rightarrow D^{* \pm}\right)$ is the probability of a $b$ quark to hadronize into a $D^{* \pm}$ meson. We adopt the value $R_{b} f\left(b \rightarrow D^{*+}\right)=(4.66 \pm 0.51) \%$ determined in Ref. [5].

We take $\alpha$ in Eq. (6) to be the running fine-structure constant, which is particularly important because it appears there in squared form. At $\sqrt{s}=10.52 \mathrm{GeV}$, we have $1 / \alpha \approx$ 132 [28]. Of course, this effect cancels out in the normalized cross sections considered in Refs. [2,3,5].

As already mentioned in Section 1, we perform for each hadron species a combined fit to the Belle, CLEO, ALEPH, and OPAL data (global fit), a separate fit to the $B$-factory data (Belle/CLEO), and a separate fit to the $Z$-factory data (ALEPH/OPAL). We perform fits both for $m \neq 0$ (GM) and for $m=0$ (ZM).

We parameterize the $z$ distributions of the $c$ and $b$ quark FFs at their starting scales $\mu_{0}$ as suggested by Bowler [29], as

$$
D_{a}^{H_{c}}\left(z, \mu_{0}\right)=N z^{-\left(1+\gamma^{2}\right)}(1-z)^{a} \mathrm{e}^{-\gamma^{2} / z},
$$

with three free parameters, $N, a$, and $\gamma$. This parameterization yielded the best fit to the Belle data [9] in a comparative analysis using the Monte-Carlo event generator JETSET/PYTHIA.

Specifically, our fitting procedure is as follows. At the scale $\mu_{f}=m_{c}=1.5 \mathrm{GeV}$, the $c$-quark FF is taken to be of the form specified in Eq. (32), while the FFs of the light quarks $q(q=u, d, s)$ and the gluon are set to zero. Then these FFs are evolved to higher scales using the DGLAP equations in Eq. (10) at NLO with $n_{f}=4$ active quark flavors and an appropriate value $\Lambda_{\overline{\mathrm{MS}}}^{(4)}$ of the asymptotic scale parameter. When the scale reaches the threshold value $\mu_{f}=m_{b}=5.0 \mathrm{GeV}$, the bottom flavor is activated and its 
Table 1: Values of fit parameters for the $D^{0}$ meson resulting from the Belle/CLEO, OPAL, and global fits in the GM approach together with the values of $\overline{\chi^{2}}$ achieved.

\begin{tabular}{|l|c|c|c|}
\hline & Belle/CLEO-GM & OPAL-GM & global-GM \\
\hline$N_{c}$ & $1.51 \cdot 10^{7}$ & $4.42 \cdot 10^{4}$ & $8.80 \cdot 10^{6}$ \\
$a_{c}$ & 1.56 & 1.52 & 1.54 \\
$\gamma_{c}$ & 3.64 & 2.83 & 3.58 \\
\hline$N_{b}$ & 13.5 & 13.5 & 78.5 \\
$a_{b}$ & 3.98 & 3.98 & 5.76 \\
$\gamma_{b}$ & 0.921 & 0.921 & 1.14 \\
\hline$\overline{\chi^{2}}$ & 3.15 & 0.794 & 4.03 \\
\hline
\end{tabular}

Table 2: Values of fit parameters for the $D^{+}$meson resulting from the Belle/CLEO, OPAL, and global fits in the GM approach together with the values of $\overline{\chi^{2}}$ achieved.

\begin{tabular}{|l|c|c|c|}
\hline & Belle/CLEO-GM & OPAL-GM & global-GM \\
\hline$N_{c}$ & $5.66 \cdot 10^{5}$ & $2.82 \cdot 10^{4}$ & $5.67 \cdot 10^{5}$ \\
$a_{c}$ & 1.15 & 1.49 & 1.16 \\
$\gamma_{c}$ & 3.39 & 2.92 & 3.39 \\
\hline$N_{b}$ & 18.8 & 18.8 & 185 \\
$a_{b}$ & 4.71 & 4.71 & 7.08 \\
$\gamma_{b}$ & 1.17 & 1.17 & 1.42 \\
\hline$\chi^{2}$ & 1.30 & 0.509 & 1.99 \\
\hline
\end{tabular}

FF is introduced in the Bowler form of Eq. (32). The evolution to higher scales is then performed with $n_{f}=5$ and the value $\Lambda_{\overline{\mathrm{MS}}}^{(5)}$ is properly matched to $\Lambda_{\overline{\mathrm{MS}}}^{(4)}$. Including $\Lambda_{\overline{\mathrm{MS}}}^{(4)}$ among the fit parameters, it turns out to be feebly constrained by the fit. Therefore, we adopt the value $\Lambda \frac{(5)}{\mathrm{MS}}=221 \mathrm{MeV}$ from Ref. [30] and adjust the value of $\Lambda_{\mathrm{MS}}^{(4)}$ accordingly, to be $\Lambda_{\frac{(4)}{\mathrm{MS}}}=321 \mathrm{MeV}$.

We first describe the Belle/CLEO fits in the GM approach. In the Belle and CLEO data, all charmed hadrons coming from $B$-meson decays are excluded, so that there is no need to include $b \rightarrow H_{c}$ fragmentation. On the other hand, the ALEPH and OPAL data each come as two sets: the sample of $H_{c}$ hadrons produced by the decays of $b$ hadrons from $Z \rightarrow b \bar{b}$ ( $b$ tagged) and the total sample of $H_{c}$ hadrons, also including those from direct production in $Z \rightarrow c \bar{c}$ and from light-quark and gluon fragmentation (total). Since we wish to test the Belle/CLEO FFs fits through comparisons to LEP1 data, we must include the $b \rightarrow H_{c}$ transitions in an appropriate way. For this purpose, we first fit the $D^{0}$ and $D^{+}$data from OPAL and the $D^{*+}$ data from ALEPH and OPAL. The resulting values of the fit parameters and of $\chi^{2}$ per degree of freedom, $\overline{\chi^{2}}$, for the $D^{0}, D^{+}$, and $D^{*+}$ mesons 
Table 3: Values of fit parameters for the $D^{*+}$ meson resulting from the Belle/CLEO, ALEPH/OPAL, and global fits in the GM approach together with the values of $\overline{\chi^{2}}$ achieved.

\begin{tabular}{|l|c|c|c|}
\hline & Belle/CLEO-GM & ALEPH/OPAL-GM & global-GM \\
\hline$N_{c}$ & $1.33 \cdot 10^{7}$ & $4.58 \cdot 10^{4}$ & $1.10 \cdot 10^{7}$ \\
$a_{c}$ & 0.992 & 1.38 & 1.07 \\
$\gamma_{c}$ & 3.84 & 3.00 & 3.81 \\
\hline$N_{b}$ & 6.67 & 6.67 & 14.0 \\
$a_{b}$ & 3.28 & 3.28 & 3.85 \\
$\gamma_{b}$ & 1.04 & 1.04 & 1.14 \\
\hline$\chi^{2}$ & 3.74 & 2.06 & 6.90 \\
\hline
\end{tabular}

are given in Tables 1 3, respectively. The goodness of these fits may also be judged from Figs. 3(a)-(c), respectively. We observe from Fig. 3(c) that the $D^{*+}$ data from ALEPH and OPAL are only moderately compatible, as was already noticed in Ref. [4. This explains why the corresponding fit has a larger value of $\overline{\chi^{2}}$ than those for the $D^{0}$ and $D^{+}$ mesons.

In a second step, we use the values of $N_{b}, a_{b}$, and $\gamma_{b}$ thus obtained as rigid input for the fits to the Belle and CLEO data. While the $b \rightarrow H_{c}$ transitions are excluded from the final states as explained above, their FFs still influence the fits through the DGLAP evolution from $\mu_{f}=m_{b}$ to $\mu_{f}=\sqrt{s}$. These fits yield new values for $N_{c}, a_{c}$, and $\gamma_{c}$, which are also included in Tables 13 together with the values of $\overline{\chi^{2}}$ achieved. The $\overline{\chi^{2}}$ values of the fits discussed so far are all acceptable, except perhaps for the fits to the $D^{0}$ and $D^{*+}$ data from Belle and CLEO, which yield $\overline{\chi^{2}}$ values in excess of 3. In Figs. 4(a)-(c), the $D^{0}, D^{+}$, and $D^{*+}$ data from Belle and CLEO are compared with the respective fit results in order to assess the goodness of the later. We observe from Figs. 4(a)-(c) that the agreement is best in the $D^{+}$case, which is also reflected in Tables 13 .

We now turn to our global fit, which uses all available $D^{0}, D^{+}$, and $D^{*+}$ data, from Belle, CLEO, ALEPH, and OPAL. The resulting values of the fit parameters and of $\overline{\chi^{2}}$ are also included in Tables 1 3. The $D^{0}, D^{+}$, and $D^{*+}$ data are compared with the respective theoretical results based on the global fit in Figs. 54 7, respectively. Comparing Figs. 547 with Figs. 3 and 4 , we find the most striking difference to be that the global fit describes the total $H_{c}$ samples from ALEPH and OPAL, especially the $D^{0}$ and $D^{+}$samples from OPAL, less well in the large- $x$ range, for $x \gtrsim 0.6$, than the ALEPH/OPAL fits. This may be understood by observing that, in the global fit, the $c \rightarrow H_{c}$ FFs, which dominantly contribute in the large- $x$ range, are mostly constrained by the more precise Belle and CLEO data and are appreciably increased in that $x$ range compared to their counterparts from the ALEPH/OPAL fits. On the other hand, the modifications of the $b \rightarrow H_{c}$ FFs are less significant and do not worsen the agreement with the ALEPH and OPAL data in 
Table 4: Values of fit parameters for the $D^{0}$ meson resulting from the Belle/CLEO, OPAL, and global fits in the ZM approach together with the values of $\overline{\chi^{2}}$ achieved.

\begin{tabular}{|l|c|c|c|}
\hline & Belle/CLEO-ZM & OPAL-ZM & global-ZM \\
\hline$N_{c}$ & $1.03 \cdot 10^{7}$ & $3.43 \cdot 10^{4}$ & $1.04 \cdot 10^{7}$ \\
$a_{c}$ & 1.48 & 1.48 & 1.50 \\
$\gamma_{c}$ & 3.60 & 2.80 & 3.60 \\
\hline$N_{b}$ & 13.4 & 13.4 & 80.8 \\
$a_{b}$ & 3.96 & 3.96 & 5.77 \\
$\gamma_{b}$ & 0.923 & 0.923 & 1.15 \\
\hline$\overline{\chi^{2}}$ & 3.25 & 0.789 & 4.66 \\
\hline
\end{tabular}

Table 5: Values of fit parameters for the $D^{+}$meson resulting from the Belle/CLEO, OPAL, and global fits in the ZM approach together with the values of $\overline{\chi^{2}}$ achieved.

\begin{tabular}{|l|c|c|c|}
\hline & Belle/CLEO-ZM & OPAL-ZM & global-ZM \\
\hline$N_{c}$ & $7.30 \cdot 10^{5}$ & $2.62 \cdot 10^{4}$ & $7.31 \cdot 10^{5}$ \\
$a_{c}$ & 1.12 & 1.48 & 1.13 \\
$\gamma_{c}$ & 3.43 & 2.91 & 3.43 \\
\hline$N_{b}$ & 19.0 & 19.0 & 163 \\
$a_{b}$ & 4.71 & 4.71 & 6.93 \\
$\gamma_{b}$ & 1.17 & 1.17 & 1.40 \\
\hline$\chi^{2}$ & 1.37 & 0.507 & 2.21 \\
\hline
\end{tabular}

any visible way.

In order to study the impact of finite $c$ and $b$ quark masses on our fits, we repeat them in the ZM approach, where $m_{c}=m_{b}=0$, except in the definition of the starting scale $\mu_{0}$. We still have $m_{H} \neq 0$, so that the $x$ distributions have finite lower endpoints and differ from the corresponding $x_{p}$ distributions in shape. The resulting values of the fit parameters and of $\overline{\chi^{2}}$ are listed for the $D^{0}, D^{+}$, and $D^{*+}$ mesons in Tables 46 , respectively. Comparing Tables 4 6 with Tables 173, we observe that the inclusion of finite quark masses reduces the $\overline{\chi^{2}}$ values of the global fits by $11-16 \%$ and also tends to reduce the $\overline{\chi^{2}}$ values of the Belle/CLEO fits, except for the case of $D^{*+}$ mesons, where the difference is insignificant. As expected, the quality of the ALEPH/OPAL fits are practically unaffected by finitequark-mass effects, which provides a retrospective justification for the use of the ZM approach in Refs. $[1,4,6,14$.

From the comparison of the fit parameters in the GM and ZM approaches presented in Tables 13 and 4,6, respectively, it is hard to judge by how much the FFs of the two approaches actually differ as functions of $z$ at a given value of $\mu_{f}$. As an example, we 
Table 6: Values of fit parameters for the $D^{*+}$ meson resulting from the Belle/CLEO, ALEPH/OPAL, and global fits in the ZM approach together with the values of $\overline{\chi^{2}}$ achieved.

\begin{tabular}{|l|c|c|c|}
\hline & Belle/CLEO-ZM & ALEPH/OPAL-ZM & global-ZM \\
\hline$N_{c}$ & $1.05 \cdot 10^{7}$ & $2.80 \cdot 10^{4}$ & $1.14 \cdot 10^{7}$ \\
$a_{c}$ & 0.929 & 1.33 & 1.03 \\
$\gamma_{c}$ & 3.82 & 2.93 & 3.82 \\
\hline$N_{b}$ & 6.52 & 6.52 & 14.9 \\
$a_{b}$ & 3.25 & 3.25 & 3.87 \\
$\gamma_{b}$ & 1.04 & 1.04 & 1.16 \\
\hline$\overline{\chi^{2}}$ & 3.69 & 2.04 & 7.64 \\
\hline
\end{tabular}

thus display in Figs. 8(a) and (b) the $z$ dependences at $\mu_{f}=10.52 \mathrm{GeV}$ of the $c \rightarrow D^{+}$ and $g \rightarrow D^{+}$FFs, respectively, of the Belle/CLEO fits in the GM and ZM approaches. We notice that there is only little difference between the GM and ZM results. This somewhat surprising observation may be understood from Fig. 9, where the ZM result for the $x_{p}$ distribution of $e^{+} e^{-} \rightarrow D^{+}+X$ at $\sqrt{s}=10.52 \mathrm{GeV}$ evaluated with the GM FFs is compared with the proper GM result. In fact, the finite- $m_{c}$ correction to the hard-scattering cross section only amounts to a few percent.

In the above implementation of the ZM approach, $m_{H}$ is identified with its physical values. It is interesting to study the impact of the finite- $m_{H}$ correction. To this end, we repeat the Belle/CLEO-ZM fit for the $D^{+}$meson putting also $m_{H}=0$, which implies that $x_{p}=x$ and $\left(\mathrm{d} \sigma / \mathrm{d} x_{p}\right)\left(x_{p}\right)=(\mathrm{d} \sigma / \mathrm{d} x)(x)$, as may be gleaned from Eq. (15). In order to obtain an acceptable value of $\overline{\chi^{2}}$, we exclude the six data points with $x_{p}<0.2$ from the fit. Furthermore, we require that $\tau>\rho_{D^{+}}$in Eq. (23), in which $\rho_{H}=0$ is put otherwise, to ensure that the hadronic energy after ISR is above the production threshold. The resulting $c \rightarrow D^{+}$and $g \rightarrow D^{+}$FFs are also shown in Figs. 8(a) and (b), respectively. We notice that the former significantly differs from its counterpart in the proper ZM approach, its peak being reduced in size and shifted to a lower value of $z$, while the latter is only moderately affected. This modification of the FFs is compensated by a reciprocal change in the line shape of the $x_{p}$ distribution, as may be seen from Fig. 9, which also contains the result of the ZM approach with $m_{H}=0$ evaluated with the Belle/CLEO-GM FFs. In fact, the peak position and height are substantially increased relative to the evaluations with $m_{H} \neq 0$. The situation is similar for the $D^{0}$ and $D^{*+}$ mesons.

Besides the $c \rightarrow H_{c}$ and $b \rightarrow H_{c}$ FFs themselves, also their first two moments are of phenomenological interest and subject to experimental determination. They correspond 
Table 7: Values of $c \rightarrow H_{c}$ and $b \rightarrow H_{c}$ branching fractions at respective production thresholds and $\mu_{f}=m_{Z}$.

\begin{tabular}{|c|c|c|c|c|c|}
\hline FF set & $H_{c}$ & $B_{c}\left(2 m_{c}\right)$ & $B_{c}\left(m_{Z}\right)$ & $B_{b}\left(2 m_{b}\right)$ & $B_{b}\left(m_{Z}\right)$ \\
\hline Belle/CLEO-GM & $D^{0}$ & 0.645 & 0.611 & 0.533 & 0.492 \\
& $D^{+}$ & 0.284 & 0.269 & 0.177 & 0.168 \\
& $D^{*+}$ & 0.262 & 0.249 & 0.219 & 0.206 \\
\hline ALEPH/OPAL-GM & $D^{0}$ & 0.629 & 0.591 & 0.533 & 0.491 \\
& $D^{+}$ & 0.234 & 0.220 & 0.177 & 0.167 \\
& $D^{*+}$ & 0.262 & 0.247 & 0.219 & 0.206 \\
\hline global-GM & $D^{0}$ & 0.642 & 0.608 & 0.526 & 0.490 \\
& $D^{+}$ & 0.283 & 0.268 & 0.166 & 0.157 \\
& $D^{*+}$ & 0.257 & 0.245 & 0.225 & 0.212 \\
\hline Belle/CLEO-ZM & $D^{0}$ & 0.656 & 0.622 & 0.530 & 0.490 \\
& $D^{+}$ & 0.288 & 0.273 & 0.177 & 0.167 \\
& $D^{*+}$ & 0.267 & 0.254 & 0.217 & 0.205 \\
\hline ALEPH/OPAL-ZM & $D^{0}$ & 0.624 & 0.587 & 0.530 & 0.489 \\
& $D^{+}$ & 0.235 & 0.221 & 0.176 & 0.166 \\
& $D^{*+}$ & 0.263 & 0.248 & 0.217 & 0.204 \\
\hline global-ZM & $D^{0}$ & 0.648 & 0.614 & 0.524 & 0.488 \\
& $D^{+}$ & 0.287 & 0.272 & 0.166 & 0.157 \\
& $D^{*+}$ & 0.260 & 0.248 & 0.222 & 0.210 \\
\hline
\end{tabular}


to the branching fractions,

$$
B_{Q}\left(\mu_{f}\right)=\int_{z_{\text {cut }}}^{1} \mathrm{~d} z D_{Q}\left(z, \mu_{f}\right)
$$

where $Q=c, b$, and the average fraction of energy that the $H_{c}$ meson receives from the $Q$ quark,

$$
\langle z\rangle_{Q}\left(\mu_{f}\right)=\frac{1}{B_{Q}\left(\mu_{f}\right)} \int_{z_{\text {cut }}}^{1} \mathrm{~d} z z D_{Q}\left(z, \mu_{f}\right),
$$

where the cut $z_{\text {cut }}=0.1$ excludes the problematic $z$ range where our formalism is not valid. As may be seen from Figs. 3 and 4 , there are no experimental data at $z<z_{\text {cut }}$ either. Tables 7 and 8 contain the values of $B_{Q}\left(\mu_{f}\right)$ and $\langle z\rangle_{Q}\left(\mu_{f}\right)$, respectively, for $Q=$ $c, b$ and $H_{c}=D^{0}, D^{+}, D^{*+}$ at the production thresholds, $\mu_{f}=2 m_{Q}$, and the $Z$-boson resonance, $\mu_{f}=m_{Z}$, for the Belle/CLEO, ALEPH/OPAL, and global fits in the GM and ZM approaches. We observe from Table 7 that the values of $B_{Q}\left(\mu_{f}\right)$ are practically independent of $\mu_{f}$, as expected. The $\mu_{f}$ dependence is likely to be mainly due our $\sqrt{s}$ independent choice of $z_{\text {cut }}$ and could probably be compensated for by using a value for $z_{\text {cut }}$ that slightly decreases with increasing value of $\sqrt{s}$. Furthermore, we find that the differences between the various sets are moderate, typically well below 10\%, except for the case of the $c \rightarrow D^{+}$transition, where the values from the OPAL fits are almost $20 \%$ below those from the Belle/CLEO fits. The results from the global fits lie between those from the Belle/CLEO and ALEPH/OPAL fits, but closer to the former. Looking at Table 8, we see that the values of $\langle z\rangle_{Q}\left(\mu_{f}\right)$ are shifted towards smaller values through the DGLAP evolution in $\mu_{f}$, as expected. As for $\langle z\rangle_{c}\left(\mu_{f}\right)$, the values from the ALEPH/OPAL fits fall 6-9\% below those from the Belle/CLEO fits, which are only slightly larger than those from the global fits. As for the ALEPH/OPAL and global fits, this trend may already be noticed by comparing Figs. 3(a), (b), and (c) with Figs. 5(b), 6(b), and 7(b), respectively. On the other hand, the differences between the various fits are marginal for $\langle z\rangle_{b}\left(\mu_{f}\right)$.

Of course, the $Q \rightarrow H_{c}$ FFs and their moments depend on scheme, order, and implementation issues such as the functional form of the ansatz at the starting scale $\mu_{0}$ and the value of $\mu_{0}$ itself, and thus do not represent physical observables by themselves. Nevertheless, comparisons of the quantities $B_{Q}\left(\mu_{f}\right)$ and $\langle z\rangle_{Q}\left(\mu_{f}\right)$ defined in Eqs. (33) and (34), respectively, with their experimental counterparts determined from the measured $x$ distributions are instructive and frequently performed in the literature [4,6,14].

\section{Conclusions}

Previous determinations of non-perturbative charmed-hadron FFs in the parton model of QCD [1,4,6, 14] were based on data from the $Z$-boson resonance, so that the effects of finite quark and hadron masses were greatly suppressed and could safely be neglected. The 
Table 8: Values of average energy fractions for $c \rightarrow H_{c}$ and $b \rightarrow H_{c}$ transitions at respective production thresholds and $\mu_{f}=m_{Z}$.

\begin{tabular}{|c|c|c|c|c|c|}
\hline FF set & $H_{c}$ & $\langle z\rangle_{c}\left(2 m_{c}\right)$ & $\langle z\rangle_{c}\left(m_{Z}\right)$ & $\langle z\rangle_{b}\left(2 m_{b}\right)$ & $\langle z\rangle_{b}\left(m_{Z}\right)$ \\
\hline Belle/CLEO-GM & $D^{0}$ & 0.641 & 0.479 & 0.308 & 0.273 \\
& $D^{+}$ & 0.648 & 0.484 & 0.334 & 0.293 \\
& $D^{*+}$ & 0.676 & 0.503 & 0.348 & 0.305 \\
\hline ALEPH/OPAL-GM & $D^{0}$ & 0.599 & 0.450 & 0.308 & 0.273 \\
& $D^{+}$ & 0.606 & 0.455 & 0.335 & 0.293 \\
& $D^{*+}$ & 0.616 & 0.462 & 0.349 & 0.305 \\
\hline global-GM & $D^{0}$ & 0.639 & 0.477 & 0.311 & 0.274 \\
& $D^{+}$ & 0.648 & 0.484 & 0.331 & 0.288 \\
& $D^{*+}$ & 0.671 & 0.499 & 0.350 & 0.306 \\
\hline Belle/CLEO-ZM & $D^{0}$ & 0.643 & 0.480 & 0.309 & 0.274 \\
& $D^{+}$ & 0.651 & 0.486 & 0.335 & 0.293 \\
& $D^{*+}$ & 0.678 & 0.504 & 0.349 & 0.306 \\
\hline ALEPH/OPAL-ZM & $D^{0}$ & 0.599 & 0.450 & 0.309 & 0.274 \\
& $D^{+}$ & 0.606 & 0.455 & 0.335 & 0.294 \\
& $D^{*+}$ & 0.614 & 0.461 & 0.349 & 0.306 \\
\hline global-ZM & $D^{0}$ & 0.642 & 0.479 & 0.312 & 0.275 \\
& $D^{+}$ & 0.651 & 0.486 & 0.331 & 0.289 \\
& $D^{*+}$ & 0.673 & 0.500 & 0.352 & 0.308 \\
\hline
\end{tabular}


advent of precise data from the $B$ factories offers us the opportunity to further constrain the charmed-hadron FFs and to test their scaling violations. However, this necessitates the incorporation of quark and hadron mass effects, which are then no longer negligible, into the formalism. The GM variable-flavor number scheme, which we previously advocated [7,12], provides a rigorous theoretical framework for this.

In this framework, we determined here new FFs for $D^{0}, D^{+}$, and $D^{*+}$ mesons through global fits to all available $e^{+} e^{-}$annihilation data, from Belle [9], CLEO [10], ALEPH [5], and OPAL [2,3]. In contrast to the situation at the $Z$-boson resonance, the $x$ distribution of the cross section for continuum production is appreciably distorted by the effects of electromagnetic ISR, which we, therefore, took into account. For comparison, we also performed fits to individual data sets. We found that the global fits somewhat suffer from the fact that the Belle and CLEO data tend to drive the average $x$ value of the $c \rightarrow H_{c}$ FFs to larger values, which leads to a worse description of the ALEPH and OPAL data. Since the $b \rightarrow H_{c}$ FFs are only indirectly constrained by the Belle and CLEO data, their form is only feebly affected by the inclusion of these data in the fits. In order to assess the significance of finite-mass effects, we repeated the fits in the ZM variable-flavor number scheme. As expected, the inclusion of finite-mass effects tends to improve the overall description of the data, by reducing the $\overline{\chi^{2}}$ values achieved. Specifically, hadron mass effects turned out to be more important than quark mass effects. In fact, they are indispensable to usefully describe the low- $x_{p}$ tails of the measured cross sections.

A FORTRAN subroutine that evaluates the FFs presented here for given values of $z$ and $\mu_{f}$ may be obtained from the authors upon request via electronic mail.

\section{Acknowledgment}

The work of T.K., B.A.K., and G.K. was supported in part by the German Federal Ministry for Education and Research BMBF through Grant No. 05 HT6GUA and by the German Research Foundation DFG through Grant No. KN 365/7-1. The work of T.K. was also supported in part by the DFG through Graduate School No. GRK 602 Future Developments in Particle Physics.

\section{A Effective electroweak charges}

The effective electroweak charges appearing in Eqs. (5), (9), (11), and (14) are given by

$$
\begin{aligned}
V_{q_{i}}^{2} & =e_{e}^{2} e_{q_{i}}^{2}+2 e_{e} v_{e} e_{q_{i}} v_{q_{i}} \rho_{1}(s)+\left(v_{e}^{2}+a_{e}^{2}\right) v_{q_{i}}^{2} \rho_{2}(s), \\
A_{q_{i}}^{2} & =\left(v_{e}^{2}+a_{e}^{2}\right) a_{q_{i}}^{2} \rho_{2}(s),
\end{aligned}
$$

where $v_{f}=\left(T_{3 f}-2 e_{f} \sin ^{2} \theta_{w}\right) /\left(2 \sin \theta_{w} \cos \theta_{w}\right)$ and $a_{f}=T_{3 f} /\left(2 \sin \theta_{w} \cos \theta_{w}\right)$ are the vector and axial-vector couplings of fermion $f$, with fractional electric charge $e_{f}$ and third 
component $T_{3 f}$ of weak isospin, to the $Z$ boson, and

$$
\begin{aligned}
& \rho_{1}(s)=\frac{s\left(s-m_{Z}^{2}\right)}{\left(s-m_{Z}^{2}\right)^{2}+m_{Z}^{2} \Gamma_{Z}^{2}}, \\
& \rho_{2}(s)=\frac{s^{2}}{\left(s-m_{Z}^{2}\right)^{2}+m_{Z}^{2} \Gamma_{Z}^{2}}
\end{aligned}
$$

are propagator functions. Here, $\theta_{w}$ is the weak mixing angle and $\Gamma_{Z}$ is the total decay width of the $Z$ boson. For small energies, $\sqrt{s} \ll m_{Z}$, the propagator functions $\rho_{1}(s)$ and $\rho_{2}(s)$ are negligible.

\section{B Single heavy-quark inclusive cross sections at $\mathcal{O}\left(\alpha_{s}\right)$}

In this appendix, we list the NLO coefficient functions appearing in Eqs. (12) and (14). We cast our results in a form similar to Ref. [16], except that our formulas include the overall factor $C_{F}$. We start by introducing the short-hand notation:

$$
\begin{aligned}
\tau_{x} & =1-x, \\
\beta_{x} & =\sqrt{1-\frac{\rho}{\tau_{x}}}, \\
\xi(x, \rho) & =\ln \frac{\rho-2 x-2 \sqrt{x^{2}-\rho}}{\rho-2 x+2 \sqrt{x^{2}-\rho}} .
\end{aligned}
$$

The coefficient functions due to virtual-soft corrections to inclusive single heavy-quark production read:

$$
\begin{aligned}
S_{T}^{(v)}(\rho)= & \frac{C_{F}}{2}\left\{( 2 - \rho ) \left[4 \ln \frac{4}{\rho} \ln \frac{1+\beta}{1-\beta}-4 \operatorname{Li}_{2}\left(-\frac{1-\beta}{2 \beta}\right)-2 \ln ^{2} \frac{2 \beta}{1-\beta}\right.\right. \\
& \left.+\frac{4}{3} \pi^{2}+\ln ^{2} \frac{1+\beta}{1-\beta}+\operatorname{Li}_{2}\left(-\frac{4 \beta}{(1-\beta)^{2}}\right)-\operatorname{Li}_{2}\left(\frac{4 \beta}{(1+\beta)^{2}}\right)\right] \\
& \left.+(10-8 \rho) \ln \frac{1+\beta}{1-\beta}-4 \beta-8 \beta \ln \frac{4}{\rho}\right\}, \\
S_{L}^{(v)}(\rho)= & C_{F}\left[\frac{\rho}{2} S_{T}^{(v)}(\rho)-\frac{\rho \beta^{2}}{2} \ln \frac{1+\beta}{1-\beta}\right], \\
S_{T}^{(a)}(\rho)= & C_{F}\left[\beta^{2} S_{T}^{(v)}(\rho)+2 \rho \beta^{2} \ln \frac{1+\beta}{1-\beta}\right], \\
S_{L}^{(a)}(\rho)= & 0 .
\end{aligned}
$$


The coefficient functions due to real corrections to inclusive single heavy-quark production read:

$$
\begin{aligned}
R_{T}^{(v)}(x, \rho)= & C_{F}\left\{\frac{2}{\sqrt{x^{2}-\rho}}\left[\rho\left(2-\tau_{x}^{2}\right)+4 \frac{\tau_{x}^{2}\left(1+\tau_{x}\right)^{3}}{\left(4 \tau_{x}+\rho\right)^{2}}+\tau_{x}\left(4+\tau_{x}\right)\left(1-\frac{2 \tau_{x}\left(1+\tau_{x}\right)}{4 \tau_{x}+\rho}\right)-2\right]\right. \\
& \left.+\frac{\rho^{2}\left(2-\tau_{x}^{2}\right)+\rho\left(2 x^{3}-7 x^{2}-1\right)+2 x^{2}\left(1+x^{2}\right)}{2\left(x^{2}-\rho\right)} \xi(x, \rho)\right\}, \\
R_{L}^{(v)}(x, \rho)= & C_{F}\left\{\frac{2}{\sqrt{x^{2}-\rho}}\left[-\rho(1-\rho)-\tau_{x}\left(\tau_{x}-2 \rho\right)+\frac{2 \tau_{x}^{2}\left(1+\tau_{x}\right)}{4 \tau_{x}+\rho}\right]\right. \\
& \left.+\frac{\rho^{3}+\rho^{2}\left(4 \tau_{x}-3\right)+\rho\left(3 x^{2}-1\right)}{2\left(x^{2}-\rho\right)} \xi(x, \rho)\right\}, \\
R_{T}^{(a)}(x, \rho)= & C_{F}\left\{\frac { 4 } { \sqrt { x ^ { 2 } - \rho } } \left[-\rho^{2}+2 \rho x+\tau_{x}^{3}+\frac{3}{2} \tau_{x}^{2}+2 \tau_{x}-1+\frac{2 \tau_{x}^{2}\left(1+\tau_{x}\right)^{3}}{\left(4 \tau_{x}+\rho\right)^{2}}\right.\right. \\
& \left.-\frac{\tau_{x}^{2}\left(1+\tau_{x}\right)\left(5 \tau_{x}+4\right)}{4 \tau_{x}+\rho}\right] \\
& \left.+\frac{-2 \rho^{3}+8 \rho^{2} x+\rho x^{2}\left(2 \tau_{x}-9\right)-\rho+2 x^{2}\left(1+x^{2}\right)}{2\left(x^{2}-\rho\right)} \xi(x, \rho)\right\}, \\
& \left.+\frac{\tau_{x}^{2} \rho\left(\rho+\tau_{x}^{2}-2\right)}{2\left(x^{2}-\rho\right)} \xi(x, \rho)\right\} .
\end{aligned}
$$

The coefficient functions due to real corrections to inclusive single gluon production read:

$$
\begin{aligned}
G_{T}^{(v)}(x, \rho)= & C_{F}\left\{2\left[\frac{1+(1-x)^{2}}{x}+\rho \frac{1-x}{x}-\frac{\rho^{2}}{2 x}\right]\left(\ln \frac{1+\beta_{x}}{1-\beta_{x}}-\beta_{x}\right)\right. \\
& \left.-4 \frac{1-x}{x} \beta_{x}-\frac{\rho^{2} \beta_{x}}{x}\right\}, \\
G_{L}^{(v)}(x, \rho)= & C_{F}\left[-\frac{2 \rho}{x} \ln \frac{1+\beta_{x}}{1-\beta_{x}}+4 \beta_{x} \frac{1-x}{x}\right], \\
G_{T}^{(a)}(x, \rho)= & C_{F}\left\{2\left[\frac{1+(1-x)^{2}}{x}-\rho \frac{1-x}{x}+\frac{\rho^{2}}{2 x}\right]\left(\ln \frac{1+\beta_{x}}{1-\beta_{x}}-\beta_{x}\right)\right. \\
& \left.-4 \frac{1-x}{x} \beta_{x}+\frac{\rho^{2} \beta_{x}}{x}\right\}, \\
G_{L}^{(a)}(x, \rho)= & C_{F}\left[\rho \frac{\rho+x^{2}+2 x-4}{x} \ln \frac{1+\beta_{x}}{1-\beta_{x}}+2 \beta_{x}(2+\rho) \frac{1-x}{x}\right] .
\end{aligned}
$$

In order to establish the subtraction terms to be included in the GM result to ensure matching with the ZM result in the massless limit, we need to take the limit $m \rightarrow 0$ 
in the virtual-soft and real correction terms listed above. In this limit, the vector and axial-vector parts coincide. Specifically, we have

$$
\begin{aligned}
\lim _{\rho \rightarrow 0}\left[S_{T}^{(u)}(\rho)+S_{L}^{(u)}(\rho)\right]= & C_{F}\left(\ln ^{2} \frac{4}{\rho}+\ln \frac{4}{\rho}-2+\pi^{2}\right) \\
\lim _{\rho \rightarrow 0} \frac{R_{T}^{(u)}(x, \rho)+R_{L}^{(u)}(x, \rho)}{(1-x)_{+}}= & C_{F}\left\{\delta(1-x)\left(-\ln ^{2} \frac{4}{\rho}+\frac{1}{2} \ln \frac{4}{\rho}-\frac{1}{2}-\frac{\pi^{2}}{3}\right)\right. \\
& +\left(\frac{1}{1-x}\right)_{+}\left[\left(1+x^{2}\right) \ln \frac{4}{\rho}-4 x+\frac{x^{2}}{2}\right] \\
& \left.-\left(1+x^{2}\right)\left[\frac{\ln (1-x)}{1-x}\right]_{+}+2 \frac{1+x^{2}}{1-x} \ln x\right\}, \\
\lim _{\rho \rightarrow 0}\left[G_{T}^{(u)}(x, \rho)+G_{L}^{(u)}(x, \rho)\right]= & 2 C_{F} \frac{1+(1-x)^{2}}{x}\left[\ln \frac{4}{\rho}+\ln (1-x)-1\right] .
\end{aligned}
$$

Comparison of Eqs. (11) and (14) with Eq. (5) yields

$$
\begin{aligned}
\lim _{\rho \rightarrow 0}\left\{\delta(1-x)\left[S_{T}^{(u)}(\rho)+S_{L}^{(u)}(\rho)\right]+\frac{R_{T}^{(u)}(x, \rho)+R_{L}^{(u)}(x, \rho)}{(1-x)_{+}}\right\}= & P_{q \rightarrow q}^{(0, T)}(x) \ln \frac{s}{\mu_{f}^{2}}+C_{q}(x) \\
& +d_{q}^{(1)}\left(x, \mu_{f}\right), \\
\lim _{\rho \rightarrow 0}\left[G_{T}^{(u)}(x, \rho)+G_{L}^{(u)}(x, \rho)\right]= & 2\left[P_{q \rightarrow g}^{(0, T)}(x) \ln \frac{s}{\mu_{f}^{2}}+C_{g}(x)\right] \\
& +d_{g}^{(1)}\left(x, \mu_{f}\right),
\end{aligned}
$$

where [16]17]

$$
\begin{aligned}
& d_{q}^{(1)}\left(x, \mu_{f}\right)=P_{q \rightarrow q}^{(0, T)}(x) \ln \frac{\mu_{f}^{2}}{m^{2}}+C_{F}\left(1+x^{2}\right)\left\{\delta(1-x)-\left(\frac{1}{1-x}\right)_{+}-2\left[\frac{\ln (1-x)}{1-x}\right]_{+}\right\}, \\
& d_{g}^{(1)}\left(x, \mu_{f}\right)=2 P_{q \rightarrow g}^{(0, T)}(x) \ln \frac{\mu_{f}^{2}}{m^{2}}-2 C_{F}(2 \ln x+1) .
\end{aligned}
$$

\section{References}

[1] B.A. Kniehl, G. Kramer, Phys. Rev. D 74 (2006) 037502 [arXiv:hep-ph/0607306].

[2] OPAL Collaboration, G. Alexander, et al., Z. Phys. C 72 (1996) 1.

[3] OPAL Collaboration, K. Ackerstaff, et al., Eur. Phys. J. C 1 (1998) 439 |arXiv:hep-ex/9708021|.

[4] J. Binnewies, B.A. Kniehl, G. Kramer, Phys. Rev. D 58 (1998) 014014 arXiv:hep-ph/9712482. 
[5] ALEPH Collaboration, R. Barate, et al., Eur. Phys. J. C 16 (2000) 597 arXiv:hep-ex/9909032.

[6] B.A. Kniehl, G. Kramer, Phys. Rev. D 71 (2005) 094013 arXiv:hep-ph/0504058.

[7] B.A. Kniehl, G. Kramer, I. Schienbein, H. Spiesberger, Phys. Rev. Lett. 96 (2006) 012001 arXiv:hep-ph/0508129.

[8] CDF Collaboration, D. Acosta, et al., Phys. Rev. Lett. 91 (2003) 241804 arXiv:hep-ex/0307080].

[9] Belle Collaboration, R. Seuster, et al., Phys. Rev. D 73 (2006) 032002 arXiv:hep-ex/0506068.

[10] CLEO Collaboration, M. Artuso, et al., Phys. Rev. D 70 (2004) 112001 arXiv:hep-ex/0402040].

[11] V.N. Gribov, L.N. Lipatov, Sov. J. Nucl. Phys. 15 (1972) 438 [Yad. Fiz. 15 (1972) 781];

G. Altarelli, G. Parisi, Nucl. Phys. B 126 (1977) 298;

Yu.L. Dokshitzer, Sov. Phys. JETP 46 (1977) 641 [Zh. Eksp. Teor. Fiz. 73 (1977) 1216].

[12] B.A. Kniehl, G. Kramer, I. Schienbein, H. Spiesberger, Phys. Rev. D 71 (2005) 014018 arXiv:hep-ph/0410289;

B.A. Kniehl, G. Kramer, I. Schienbein, H. Spiesberger, Eur. Phys. J. C 41 (2005) 199 [arXiv:hep-ph/0502194];

B.A. Kniehl, G. Kramer, I. Schienbein, H. Spiesberger, Report No. DESY 07-066, MZ-TH/07-07, and LPSC 07-46 arXiv:0705.4392 [hep-ph]], to appear in Phys. Rev. D.

[13] R. Baier, K. Fey, Z. Phys. C 2 (1979) 339;

G. Altarelli, R.K. Ellis, G. Martinelli, S.-Y. Pi, Nucl. Phys. B 160 (1979) 301.

[14] J. Binnewies, B.A. Kniehl, G. Kramer, Z. Phys. C 76 (1997) 677 arXiv:hep-ph/9702408.

[15] J. Binnewies, B.A. Kniehl, G. Kramer, Z. Phys. C 65 (1995) 471 arXiv:hep-ph/9407347];

J. Binnewies, B.A. Kniehl, G. Kramer, Phys. Rev. D 53 (1996) 3573 arXiv:hep-ph/9506437;

B.A. Kniehl, G. Kramer, B. Pötter, Nucl. Phys. B 582 (2000) 514 arXiv:hep-ph/0010289;

B.A. Kniehl, G. Kramer, B. Pötter, Phys. Rev. Lett. 85 (2000) 5288 arXiv:hep-ph/0003297];

B.A. Kniehl, G. Kramer, B. Pötter, Nucl. Phys. B 597 (2001) 337 
arXiv:hep-ph/0011155;

S. Albino, B.A. Kniehl, G. Kramer, Nucl. Phys. B 725 (2005) 181 arXiv:hep-ph/0502188;

S. Albino, B.A. Kniehl, G. Kramer, Nucl. Phys. B 734 (2006) 50 arXiv:hep-ph/0510173];

M. Hirai, S. Kumano, T.H. Nagai, K. Sudoh, Phys. Rev. D 75 (2007) 094009 arXiv:hep-ph/0702250;

D. de Florian, R. Sassot, M. Stratmann, Phys. Rev. D 75 (2007) 114010 arXiv:hep-ph/0703242.

[16] P. Nason, B.R. Webber, Nucl. Phys. B 421 (1994) 473;

P. Nason, B.R. Webber, Nucl. Phys. B 480 (1996) 755, Erratum.

[17] B. Mele, P. Nason, Nucl. Phys. B 361 (1991) 626;

J.P. Ma, Nucl. Phys. B 506 (1997) 329 arXiv:hep-ph/9705446].

[18] S. Albino, B.A. Kniehl, G. Kramer, W. Ochs, Phys. Rev. D 73 (2006) 054020 arXiv:hep-ph/0510319.

[19] B.A. Kniehl, L. Zwirner, Phys. Lett. B 468 (1999) 294 arXiv:hep-ph/9909517.

[20] M. Cacciari, P. Nason, C. Oleari, J. High Energy Phys. 04 (2006) 006 arXiv:hep-ph/0510032.

[21] E.A. Kuraev, V.S. Fadin, Sov. J. Nucl. Phys. 41 (1985) 466 [Yad. Fiz. 41 (1985) 733]; G. Altarelli, G. Martinelli, in Physics at LEP, edited by J. Ellis and R. Peccei, CERN Yellow Report No. 86-02, Volume 1, 1986, p. 47;

O. Nicrosini, L. Trentadue, Z. Phys. C 39 (1988) 479;

F.A. Berends, W.L. van Neerven, G.J.H. Burgers, Nucl. Phys. B 297 (1988) 429;

F.A. Berends, W.L. van Neerven, G.J.H. Burgers, Nucl. Phys. B 304 (1988) 921, Erratum;

W. Beenakker, F.A. Berends, S.C. van der Marck, Nucl. Phys. B 349 (1991) 323.

[22] O. Nicrosini, L. Trentadue, Phys. Lett. B 196 (1987) 551.

[23] V.N. Gribov, L.N. Lipatov, Sov. J. Nucl. Phys. 15 (1972) 675 [Yad. Fiz. 15 (1972) 1218].

[24] R. Seuster (private communication).

[25] Particle Data Group, L. Montanet, et al., Phys. Rev. D 50 (1994) 1173.

[26] Particle Data Group, R.M. Barnett, et al., Phys. Rev. D 54 (1996) 1.

[27] Particle Data Group, C. Caso, et al., Eur. Phys. J. C 3 (1998) 1. 
[28] K. Hagiwara, S. Matsumoto, D. Haidt, C.S. Kim, Z. Phys. C 64 (1994) 559 [arXiv:hep-ph/9409380];

K. Hagiwara, S. Matsumoto, D. Haidt, C.S. Kim, Z. Phys. C 68 (1995) 352, Erratum.

[29] M.G. Bowler, Z. Phys. C 11 (1981) 169.

[30] Particle Data Group, W.M. Yao, et al., J. Phys. G 33 (2006) 1. 


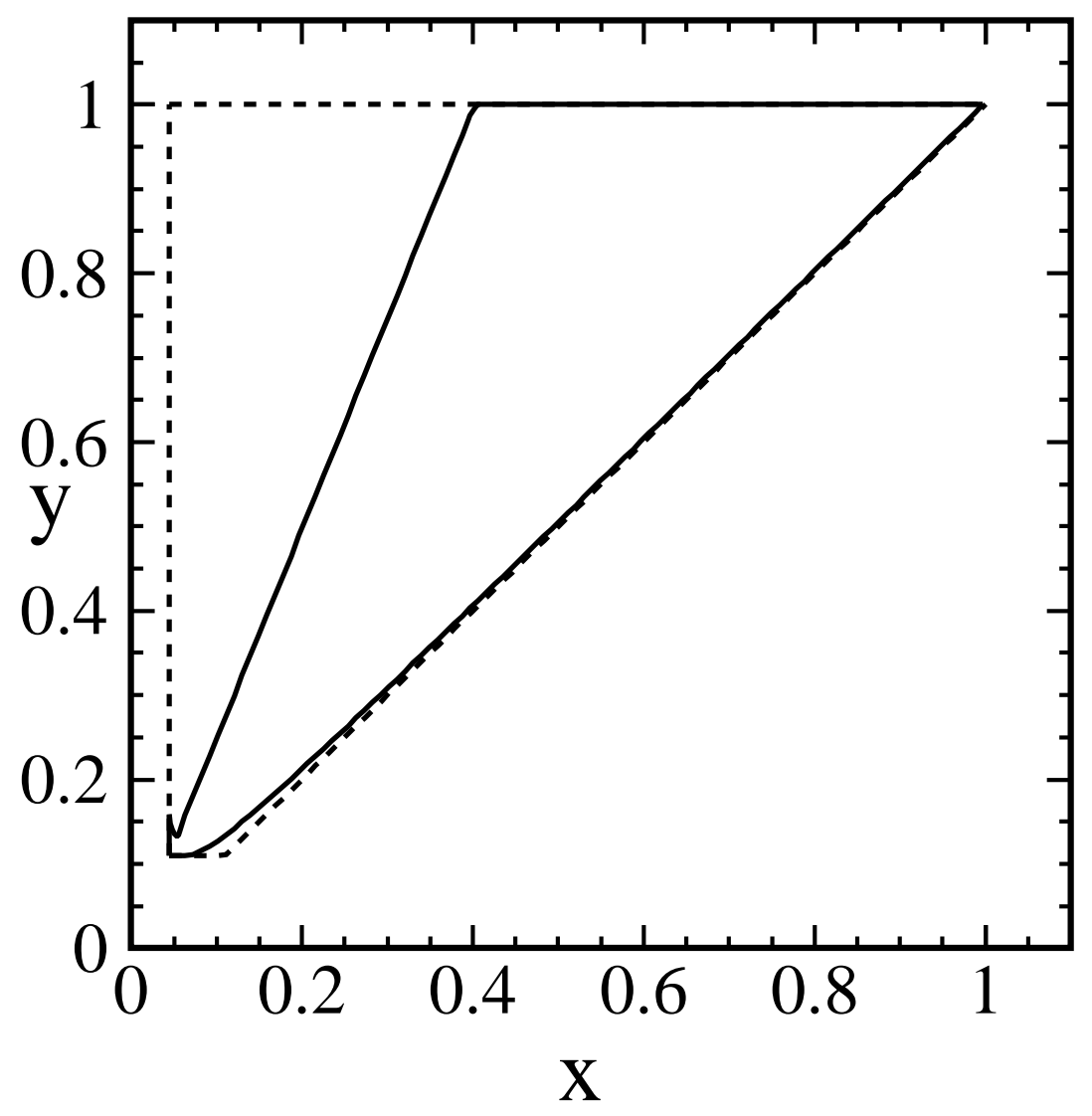

Figure 1: Kinematically accessible region in the $(x, y)$ plain for $e^{+} e^{-} \rightarrow b+X$ and subsequent decay $b \rightarrow H_{c}+X$ with collinear emission of $H_{c}$ assuming $\sqrt{s}=m_{Z}, m=$ $5.0 \mathrm{GeV}$, and $m_{H}=2.01 \mathrm{GeV}$ (solid line). For comparison, also the region used in our analysis is shown (dashed line). 


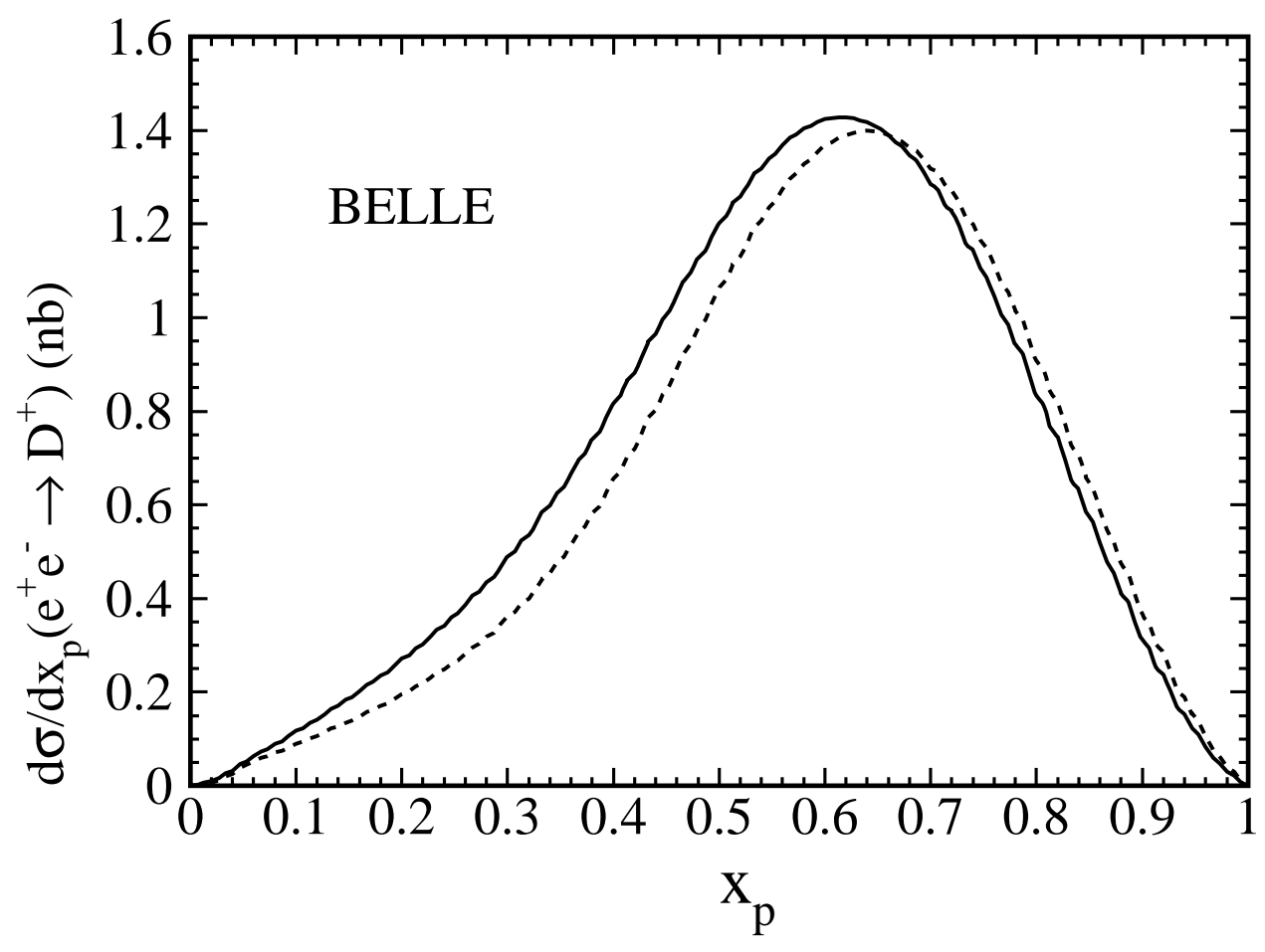

Figure 2: $x_{p}$ distributions of $e^{+} e^{-} \rightarrow D^{+}+X$ at $\sqrt{s}=10.52 \mathrm{GeV}$ evaluated in the GM approach with the FFs from the joint fit to the Belle [9] and CLEO [10] data including ISR corrections (solid line) and corresponding result with the latter subtracted (dashed line). 


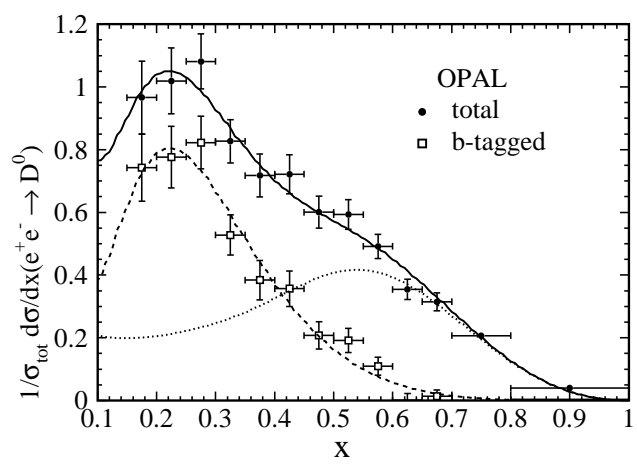

(a)

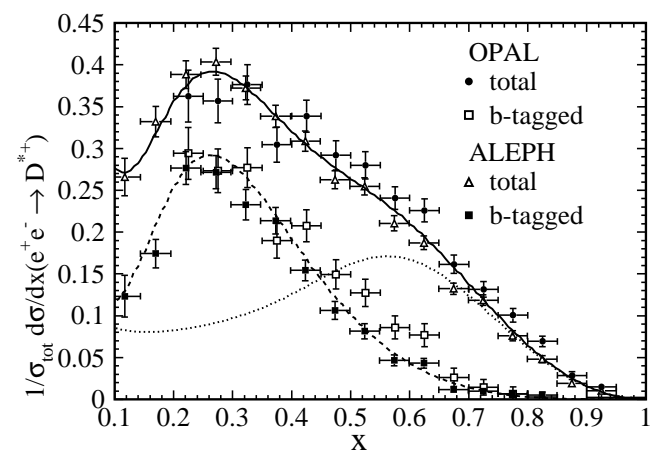

(c)

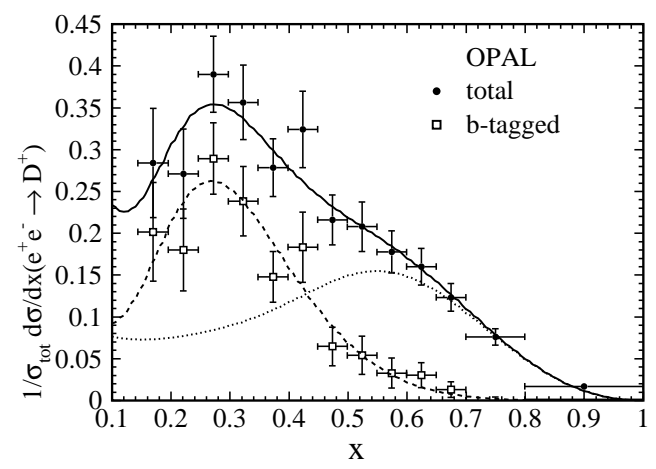

(b)

Figure 3: Normalized $x$ distributions of (a) $D^{0}$, (b) $D^{+}$, and (c) $D^{*+}$ mesons from OPAL [2,3] and ALEPH [5] compared to the respective fits in the GM approach from Tables 1, 3. The dotted, dashed, and solid lines refer to the $c$-quark-initiated, $b$-quark-initiated, and total contributions, respectively. 


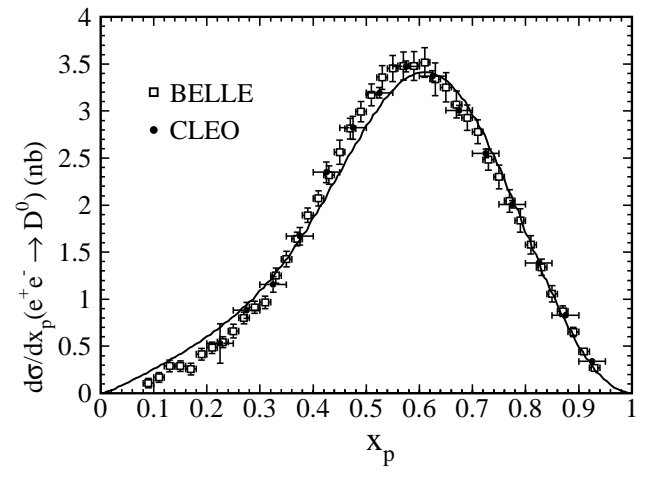

(a)

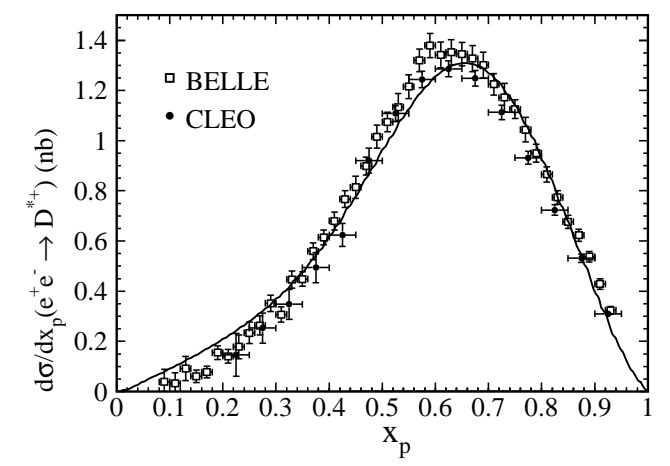

(c)

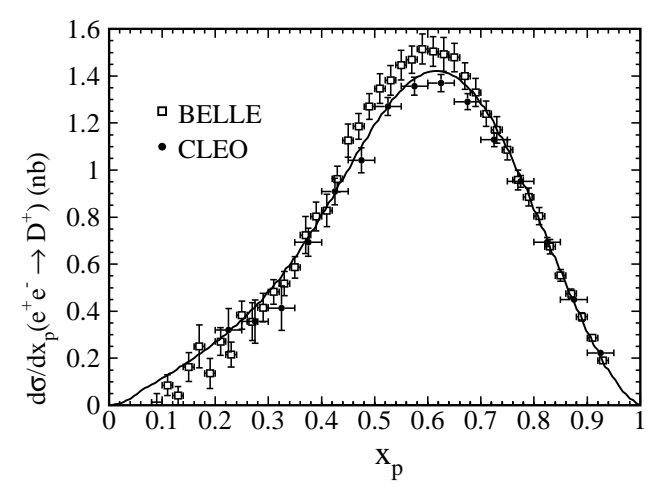

(b)

Figure 4: $x_{p}$ distributions of (a) $D^{0}$, (b) $D^{+}$, and (c) $D^{*+}$ mesons from Belle [9] and CLEO [10] compared to the respective fits in the GM approach from Tables 13 . 3 . 


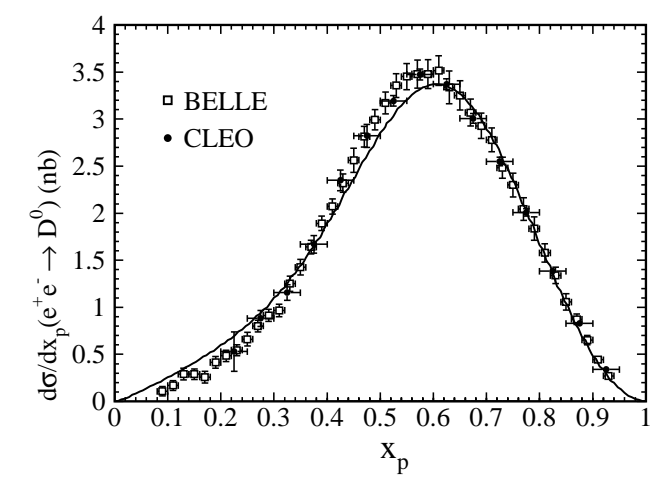

(a)

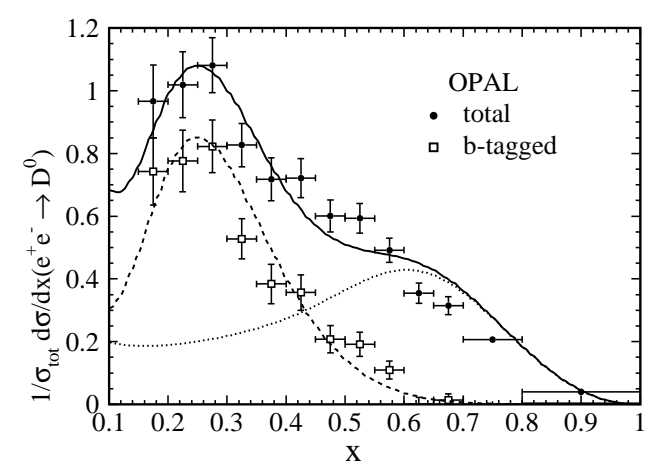

(b)

Figure 5: $x_{p}$ distributions of $D^{0}$ mesons from (a) Belle [9] and CLEO [10], and (b) normalized $x$ distribution of $D^{0}$ mesons from OPAL 2] compared to the global fit from Table 1. In frame (b), the dotted, dashed, and solid lines refer to the $c$-quark-initiated, $b$-quark-initiated, and total contributions, respectively.

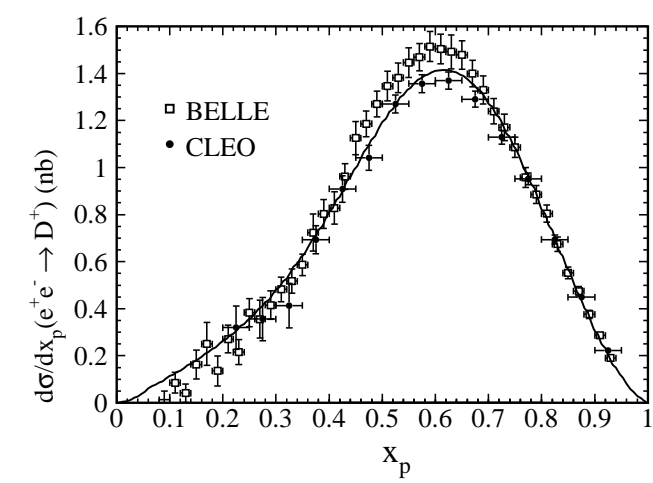

(a)

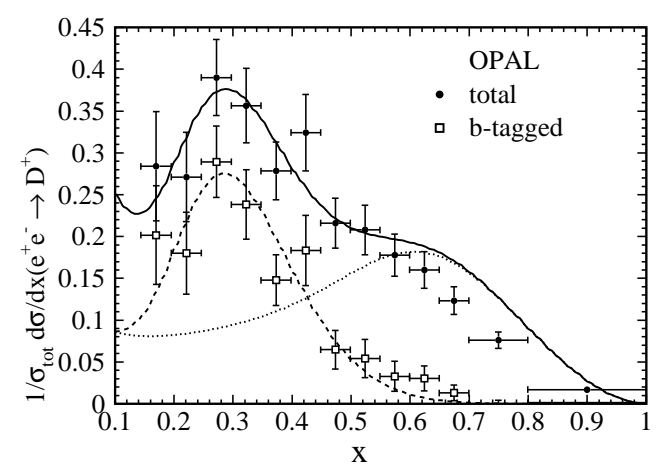

(b)

Figure 6: $x_{p}$ distributions of $D^{+}$mesons from (a) Belle [9] and CLEO [10], and (b) normalized $x$ distribution of $D^{+}$mesons from OPAL [2] compared to the global fit from Table 2. In frame (b), the dotted, dashed, and solid lines refer to the $c$-quark-initiated, $b$-quark-initiated, and total contributions, respectively. 


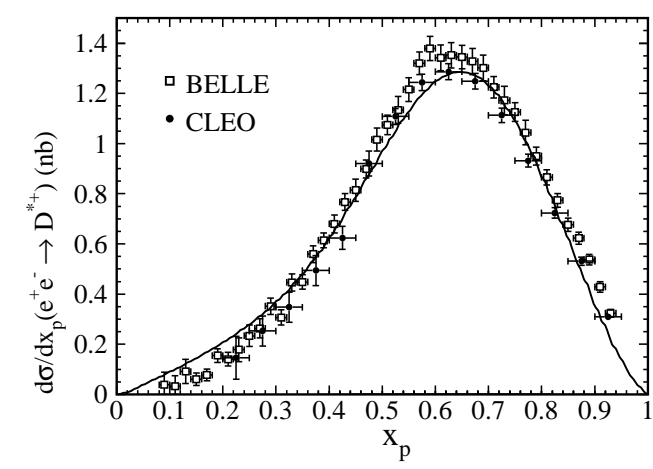

(a)

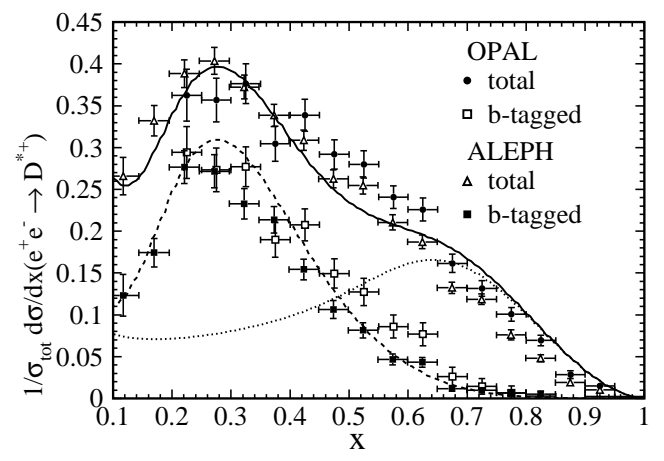

(b)

Figure 7: $x_{p}$ distributions of $D^{*+}$ mesons from (a) Belle [9] and CLEO [10], and (b) normalized $x$ distributions of $D^{*+}$ mesons from ALEPH [5], and OPAL [3] compared to the global fit from Table 3. In frame (b), the dotted, dashed, and solid lines refer to the $c$-quark-initiated, b-quark-initiated, and total contributions, respectively.

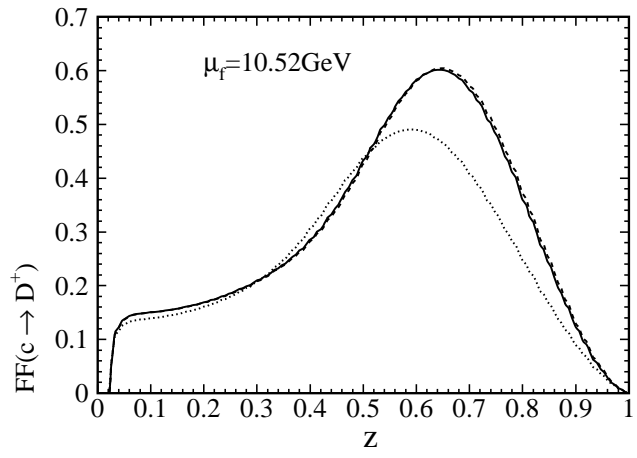

(a)

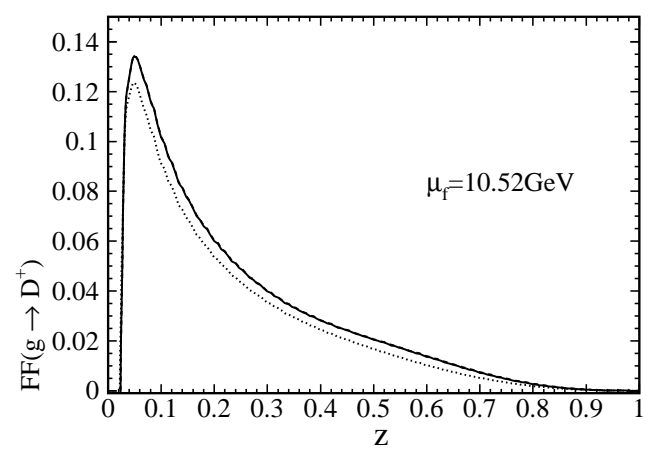

(b)

Figure 8: (a) $c \rightarrow D^{+}$and (b) $g \rightarrow D^{+}$FFs of the Belle/CLEO fits at $\mu_{f}=10.52 \mathrm{GeV}$ as functions of $z$ in the GM approach (solid lines) and in the ZM approaches with $m_{H} \neq 0$ (dashed lines) and $m_{H}=0$ (dotted lines). 


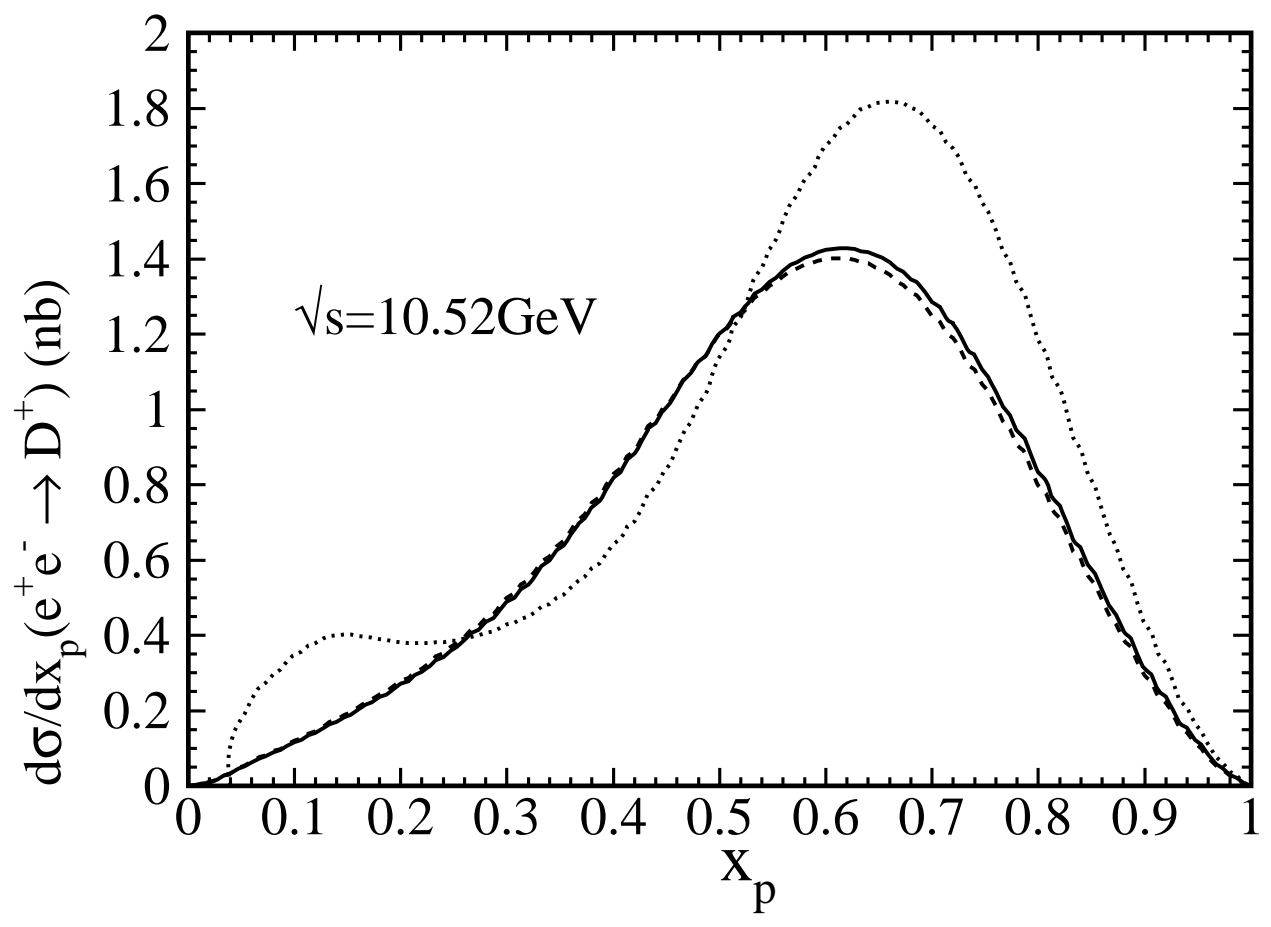

Figure 9: $x_{p}$ distributions of $e^{+} e^{-} \rightarrow D^{+}+X$ at $\sqrt{s}=10.52 \mathrm{GeV}$ in the GM approach (solid line) and in the ZM approaches with $m_{H} \neq 0$ (dashed lines) and $m_{H}=0$ (dotted lines), all evaluated with the FFs from the Belle/CLEO-GM fit. 